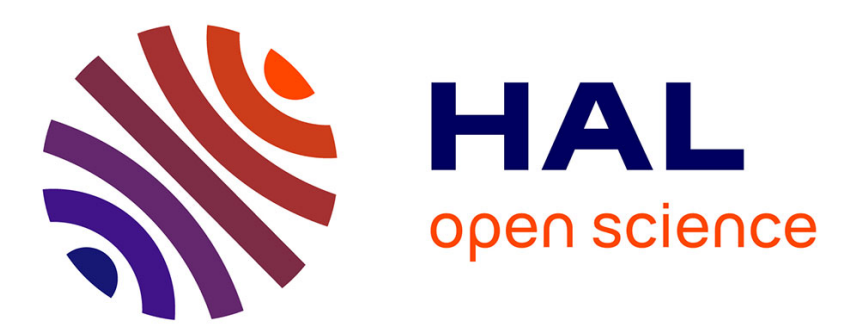

\title{
Iterations of the sawtooth map as a dynamical model for CVD/CVI SiC polytype growth
}

\author{
Gerard L. Vignoles
}

\section{To cite this version:}

Gerard L. Vignoles. Iterations of the sawtooth map as a dynamical model for CVD/CVI SiC polytype growth. International journal of bifurcation and chaos in applied sciences and engineering , 1993, 3, pp.1177-1194. 10.1142/S0218127493000970 . hal-00327300

\section{HAL Id: hal-00327300 \\ https://hal.science/hal-00327300}

Submitted on 16 Oct 2008

HAL is a multi-disciplinary open access archive for the deposit and dissemination of scientific research documents, whether they are published or not. The documents may come from teaching and research institutions in France or abroad, or from public or private research centers.
L'archive ouverte pluridisciplinaire HAL, est destinée au dépôt et à la diffusion de documents scientifiques de niveau recherche, publiés ou non, émanant des établissements d'enseignement et de recherche français ou étrangers, des laboratoires publics ou privés. 


\title{
Iterations of the sawtooth map as a dynamical model for CVD/CVI SiC polytype growth
}

\author{
G. L. VIGNOLES \\ Laboratoire des Composites ThermoStructuraux \\ 3, Allée La Boëtie - Université Bordeaux 1 \\ F33600 PESSAC, France \\ vinhola@1cts.u-bordeaux1.fr
}

Published in

International Journal of Bifurcation and Chaos, vol. 3 (1993), pp. 1177-1194. 


\title{
Iterations of the Sawtooth Map as a Dynamical Model for CVD/CVI SiC Polytype Growth
}

\author{
Gérard L. Vignoles \\ Laboratoire des Composites Thermostructuraux (UMR 47 CNRS-SEP-UB1), \\ Domaine Universitaire, 3, Allée La Boëtie, F33600 Pessac
}

\begin{abstract}
.
In order to describe silicon carbide polytype growth in CVD/CVI conditions, a dynamical model based on the iterations of the sawtooth map has been studied. A two-parameter bifurcation diagram has been computed for a simple logistic-like family, and its structure has been studied in more detail by means of kneading theory and study of the skeleton. All the routes to chaos are shown to derive from the application of a central theorem involved in the construction of the skeleton. Comparison, under a combinatorial point of view, between this mathematical model and the reported data on known $\mathrm{SiC}$ polytypes is achieved and discussed. Indeed, the model is able to generate any periodic or chaotic polytypic sequence.
\end{abstract}


The Sawtooth Map and the growth of SiC polytypes

\section{Introduction.}

The study of silicon carbide polytypism in far-fromequilibrium growth conditions, such as CVD and CVI from halogenosilanehydrogen mixtures, has recently led to the formulation of a dynamical model based on the iterations of a one-dimensional mapping [Vignoles, 1992]. The aim of the present contribution is to analyse on a more theoretical point of view the properties of this dynamical system.

Using experimental data on the deformations of atomic Si-C bilayers [Cheng et al., 1990] and linking them with their relative orientations, a relation has been found between the relative deformation $\delta_{n}$ $=\left(\mathrm{L}_{n}-\mathrm{l}_{n}\right) \mathrm{l}_{n}\left(\mathrm{~L}_{n}\right.$ and $\mathrm{l}_{n}$ being respectively the interlayer and intralayer bond lengths) of any layer $n$, and the deformation $\delta_{n+1}$ of the next layer $n+1$, as a function $f: \delta_{n} \mapsto \delta_{n+1}$, whose plot is shown in Fig. 1. Its shape is characteristic : there is a critical value $\delta^{*}$ in the interval so that the function is continuous and increasing in both of the subintervals defined by $\delta^{*}$. The discontinuity at $\delta^{*}$ makes the graph look like a sawtooth, so the name "sawtooth map" will be used to refer to such an endomorphism. Each of the two subintervals is related to one of the two possible orientations of the bilayer (namely, $\mathbf{h}$ and $\mathbf{k}$ for hexagonal and cubic packing), and the iteration of the mapping generates orientation sequences deduced from the position of the variable $\delta_{n}$ relatively to $\delta^{*}$. According to the particular shape of the graph of $f$, these sequences may converge to some steady state, or to a periodic regime, or exhibit a chaotic behavior, a fact that accounts qualitatively for the diversity of SiC polytypism [Verma \& Krishna, 1966 ; Fisher \& Barnes, 1990].

It is of a particular interest to characterize these orientation suites, to classify them, to compare them with the suites experimentally encountered in the polytypes of $\mathrm{SiC}$ or other similar materials, to relate 
The Sawtooth Map and the growth of SiC polytypes

them with control parameters, and to prove the onset of chaos, so as to account for the existence of both periodic and chaotic polytypes of SiC. This can only be made through a better understanding of the mathematical properties of the model.

To do so, a study will first be made on a "model family" of sawtooth maps, in order to recognize all the possible permanent regimes obtained by variations of the shape of the plot. This will be done by drawing a two-parameter bifurcation map of the model family. Secondly, it will be attempted to highlight the combinatorial properties of this family and to show to which extent they are common to all the sawtooth maps, by means of the algebra of the itineraries and of the kneading theory [Milnor \& Thurston, 1988]. The main results of this part are that (i) any sequence occurs by a two-parameter variation of the function, and (ii) a boundary for topological chaos is determined. A comparison with the reality of polytypism is then made.

\section{Study of a Model Family of Sawtooth Maps.}

\subsection{Definition of the model family.}

For sake of simplicity, and without loss of generality, an example family of functions will be studied, with a particularly simple mathematical expression, inspired from the well-known logistic function [May, 1976], and aiming at having a complete family, as defined by [Collet \& Eckmann, 1980]. Its expression is the following :

$$
\begin{aligned}
f_{\lambda_{1}, \lambda_{2}}:[0,1] & \rightarrow[0,1] \\
x & \mapsto\left\{\begin{array}{ll}
f_{\mathrm{L}}(x)=4 \lambda_{1} x(1-x) & \text { if } x \leq 0.5 \\
f_{\mathrm{R}}(x)=1-4 \lambda_{2} x(1-x) & \text { if } x>0.5
\end{array} \quad, \lambda_{1} \text { and } \lambda_{2} \in[0,1]\right.
\end{aligned}
$$

As the expression in (1) depends on two parameters, $\lambda_{1}$ and $\lambda_{2}$, it is said to define a 2-parameter family of mappings of the unit segment 
The Sawtooth Map and the growth of SiC polytypes

$[0,1]$. The plot of $f_{\lambda_{1}, \lambda_{2}}$ is made of two parabolic curves of opposite curvature, as shown in Fig. 2. The parameters monitor the height of these curves in an independent but symmetric fashion.

In order to modelize analog-to-digital signal converters, functions of the class of $f_{\lambda_{1}, \lambda_{2}}$ have already been studied in [Leonov, 1959 \& 1960] and [Mira, 1978], namely :

$$
\mathrm{U}_{v, \lambda_{1}, \lambda_{2}}: \quad x \mapsto \begin{cases}T_{1}(x)=v+\lambda_{1} x & \text { if } x<0 \\ T_{2}(x)=-1+\lambda_{2} x & \text { if } x>0\end{cases}
$$

The three-parameter study of this family has led to introduce the notion of degree of complexity of itineraries [Fournier-Prunaret, 1991], which will be used in the discussion.

Another study, in [Feely \& Chua, 1992], concerning the following function :

$$
\mathrm{U}_{\lambda_{1}, \lambda_{2}}: \quad x \mapsto \lambda_{1} x+\lambda_{2}-\operatorname{sgn}(x)
$$

where $\lambda_{1}>1$ has been performed in order to complete Leonov's results. A complete discussion of the admissibility of periodic (unstable) itineraries in the $\left(\lambda_{1}, \lambda_{2}\right)$ parameter space has been given.

From an initial value, the function $f_{\lambda_{1}, \lambda_{2}}$ allows to generate a suite $x_{1}=f\left(x_{0}\right), x_{2}=f\left(x_{1}\right), x_{3}=f\left(x_{2}\right), \quad, x_{n+1}=f\left(x_{n}\right)$ : The set $\left\{x_{0}, x_{1}, x_{2}, \ldots x_{n}, \ldots\right\}$ is the trajectory of $x_{0}$ under the action of $f_{\lambda_{1}, \lambda_{2}}$. The iteration of $f$ constitutes a discrete dynamical system, in which a variable evolves with time, here represented by the integer index $n$.

It has already been pointed out that the left part of $f$ corresponds to the orientation $\mathbf{h}$, and the right part to $\mathbf{k}$; thus, a trajectory has an underlying $\mathbf{h}-\mathbf{k}$ sequence. This suits well the mathematical definition of itineraries [Sharkovskii, 1964 ; Stefan, 1977 ; Metropolis et 
The Sawtooth Map and the growth of SiC polytypes

al., 1973]. Let us recall first the definition of an itinerary suitable to this case : at each iteration, the position of $x_{n}$ is considered in relation with the discontinuity $x^{*}$ and a letter $\mathrm{U}_{n}$ is attributed in each case :

$$
\begin{aligned}
& \mathrm{U}_{n}=\mathrm{L} \text { if } x_{n} \in\left[0, x^{*}[;\right. \\
& \mathrm{U}_{n}=\mathrm{C} \text { if } x_{n}=x^{*} ; \\
& \left.\left.\mathrm{U}_{n}=\mathrm{R} \text { if } x_{n} \in\right] x^{*}, 1\right] .
\end{aligned}
$$

The $\mathrm{U}_{n}$ suite is called itinerary of the point $x_{0}$. From a physical point of view, the letters $L$ and $R$ match respectively with the orientations $\mathbf{k}$ and $\mathbf{h}$, and the letter $\mathrm{C}$ is physically unlikely to occur. Other conventions have already been used to describe itineraries. Leonov [1960] uses directly the expression $T_{1}$ when the left hand of the graph is used and $T_{2}$ for the converse case. Many authors [e. g., Feely \& Chua, 1992 ; Isola \& Politi, 1990] use the numbers 0 for left and 1 for right, a convention which leads to the build-up of a sequence algrebra, as shown in later sections.

\subsection{Discussion of the two-parameter bifurcation diagram.}

Attention will be only paid to the permanent part of the trajectories, since the phenomenon intended to be accounted for is a crystal layer-by-layer growth on a possibly large number of layers.

\subsubsection{Computer simulation.}

It is possible, by means of an extremely simple computer program, to sketch the long-time behavior of the mapping according to the two parameter values. Any mapping will be represented as a point in a discretized $\left(\lambda_{1}, \lambda_{2}\right)$ parameter plane. For each couple of parameters, a large number of initial iterations are performed. An arbitrary initial value of 0.3 has been taken for all calculations. Then, periodicity is detected on a sample trajectory up to some limit. As the aim is to deal with orientation sequences, 
The Sawtooth Map and the growth of SiC polytypes

a parallel production of such sequences is produced by the computer and used to determine periodicity through an autocorrelation criterion computation. Consequently, this method does not focus on the true periodicity of the mappings, which can be different from the periodicity of the derived sequences. Associating a color to the period $p$ found for each point from a 158-color palette, one obtains the two-parameter bifurcation diagram shown in Fig. 3a. At first sight, some remarks have to be made.

First of all, the diagram exhibits axial symmetry relative to the first diagonal of equation $\lambda_{1}=\lambda_{2}$, which is not surprising in relation to the symmetric role of the two parameters. Secondly, the diagram is neatly divided in two parts : one steady-state (period 1) part for $\lambda_{1}<0.5$ or $\lambda_{2}<0.5$, and a very complicated part consisting of points of periodic $(1<\mathrm{p} \leq 156)$ and "aperiodic" ( $p>156)$ behavior.

\subsubsection{Steady-state behavior.}

The steady-state regimes correspond to the existence of attractive points on the graph. For example, if $\lambda_{1}<0.5$, there exists an $x \in$ $[0,0.5]$ so that $f(x)=x$ and that $\left|f^{\prime}(x)\right|<1$, as depicted in Fig. 4a. The iterates of $f$ tend to converge towards this point. When both $\lambda_{1}$ and $\lambda_{2}$ are inferior to 0.5 , two stable attractors coexist, each one attracting the points in its respective basin of $[0,1]$.

The loss of stability for the steady states occur when any of the two parameters gets superior to 0.5 . For example, when $\lambda_{1}$ passes through this value, the intersection of the first part of the curve and the diagonal (with $\left|f_{\lambda_{1}, \lambda_{2}}\right|<1$ ) collides with the discontinuity and then disappears.

\subsubsection{Direct and inverse cascades.}

The most interesting part of the graph is obviously the most complicated one, located in the square $[0.5,1] \times[0.5,1]$, illustrated in Fig. $3 \mathrm{~b}$. 
The largest periodic zone is a fish-like symmetrical zone made of a body and two tails, further called a "fish" for sake of abbreviation. This zone corresponds to stable period- 2 regimes, of repeated sequence $\mathbf{h k}$, as illustrated in Fig. 4b. The boundaries of the body of this fish correspond to the crossing of one of the two periodic points through the value 0.5. As a matter of fact, the exact analytical expression of these curves can be found by solving the following equations :

$$
\begin{aligned}
& f^{2}\left(1 / 2^{-}\right)=1 / 2 \\
& f^{2}\left(1 / 2^{+}\right)=1 / 2
\end{aligned}
$$

Eq. (5a) and (5b) mean that a two-period cycle collides into the discontinuity respectively by the left side and by the right side. The expression of $f^{2}(x)$ is the following :

$$
\begin{aligned}
& f^{2}(x)=\left\{\begin{array}{cc}
4 \lambda_{1} f(x)(1-f(x)) & \text { if } f(x)<1 / 2 \\
1-4 \lambda_{2} f(x)(1-f(x)) & \text { if } f(x)>1 / 2
\end{array}\right. \\
& = \begin{cases}16 \lambda_{1}^{2} x(1-x)\left(1-4 \lambda_{1} x(1-x)\right) & \text { if } f(x)<1 / 2 \text { and } x<1 / 2 \\
1-16 \lambda_{1} \lambda_{2} x(1-x)\left(1-4 \lambda_{1} x(1-x)\right) & \text { if } f(x)>1 / 2 \text { and } x<1 / 2 \\
16 \lambda_{1} \lambda_{2}\left(1-4 \lambda_{2} x(1-x)\right) x(1-x) & \text { if } f(x)<1 / 2 \text { and } x>1 / 2 \\
1-16 \lambda_{2}^{2}\left(1-4 \lambda_{2} x(1-x)\right) x(1-x) & \text { if } f(x)>1 / 2 \text { and } x>1 / 2\end{cases}
\end{aligned}
$$

$1 / 2^{-}$belongs to the second case ; the resolution is then very simple :

$$
\begin{aligned}
& f(1 / 2)=1 / 2 \\
\Leftrightarrow & 1-16 \lambda_{1} \lambda_{2} \times 1 / 4\left(1-4 \lambda_{1} \times 1 / 4\right)=1 / 2 \\
\Leftrightarrow & 1-4 \lambda_{1} \lambda_{2}\left(1-\lambda_{1}\right)=1 / 2 \\
\Leftrightarrow & 4 \lambda_{1} \lambda_{2}\left(1-\lambda_{1}\right)=1 / 2 \\
\Leftrightarrow & \lambda_{2}=\frac{1}{8 \lambda_{1}\left(1-\lambda_{1}\right)}
\end{aligned}
$$

In the same way, (5b) leads to :

$$
\lambda_{1}=\frac{1}{8 \lambda_{2}\left(1-\lambda_{2}\right)}
$$


The Sawtooth Map and the growth of SiC polytypes

(7a) and (7b) are the equations of two hyperbolic-like curves in the $\left(\lambda_{1}, \lambda_{2}\right)$ plane limiting the body of the period-2 fish. This calculation shows the critical importance of the trajectories of the critical points $1 / 2^{-}$ and $1 / 2^{+}$. Indeed, they will be used in a further section for the combinatorial study of the bifurcations.

On each side of the period-2 zone are located other fish-like zones of period $3,4,5,6$, etc... building up a direct cascade of bifurcations when one of the parameters is increased. As the sequences series are hk, hkk, hkkk, ... and hk, hhk, hhhk, ..., they are the exact equivalent of Mira's "boxes" of first degre of complexity in the "boxes-in-files" bifurcation structure described for Leonov's mapping [Gumowski \& Mira, 1980].

A closer glance permits to see that between any of the fishes, there are smaller ones, whose period is the sum of its two bigger neighbors, e. g. a period 5 fish is located between fishes of period 2 and 3 . This remark may be translated into a "fish reproduction rule" [Leonov, 1959] :

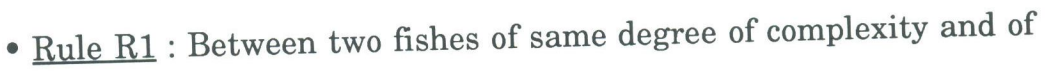
period $p$ and $q$, there always exists a fish of period $(p+q)$.

An illustration of $\mathrm{R} 1$ is shown in Fig. 5a. More precisely, R1 can be expressed by means of a "+" operation between two fishes of the same complexity :

$$
(\mathrm{RL})^{\infty}+(\mathrm{RLL})^{\infty}=(\mathrm{RLRLL})^{\infty}
$$

This relation may be translated using the traditional polytype notations :

- h-k notation [Jagodzinski, 1949] : hk + hkk = hkhkk

$$
\text { - Zhdanov notation [Zhdanov, 1945] : (2) + (3) = (23) }
$$


As a matter of fact, the period-2 fish lies between two "period1 " (i.e. steady-state) zones and is itself a consequence of that construction rule, but applied to "fishes" of degree 0. Moreover, between period 2 and period 1 , one finds a period $2+1=3$, between 3 and 1 , there is a fish of period $3+1=4$, and so on... : the direct cascade is deduced from rule R1.

Looking closer at the disposition of the fishes between the period-2 and period-5 ones, the following order can be seen : 2-...-13-11-9-75 , which corresponds to an inverse cascade. So rule R1 gives rise to both direct and inverse cascades, which are indeed intricate in a recursive fashion : it is always possible to find an inverse cascade between two terms of a given direct cascade, and to find a direct cascade between two terms of an inverse one. Some consequences appear :

- Any fish body is an accumulation locus for direct and inverse cascades on both sides [Leonov, 1959].

- Two fishes of a same degree of complexity are disjointed [Leonov, 1959].

- The intrication of cascades is in some sense equivalent to the structure of the devil's staircase [Feely \& Chua 1992].

This gives a larger understanding of the similar recent results obtained for the "gap map" [De Souza Vieira et al., 1987 ; Chia \& Tan, 1992].

All the fishes constructed by means of rule $\mathrm{R} 1$ have their nose in common. For example, the period- $2,-3,-4$, etc... fishes already mentioned share a common nose at $(0.5,0.5)$. It can be said that a complete "boxes in files" bifurcation structure hangs to this nose. 


\subsubsection{Subharmonic cascades.}

Between the two tails of the period-2 fish lies a smaller one of period 4, then another still smaller of period 8, and so on.... These fishes accumulate along the first diagonal up to a point where $\lambda_{1}=\lambda_{2}=\lambda(\infty)<1$. Past this point, no periodicity is detected. This phenomenon is a subharmonic cascade. Its mechanism is different of the cascade first described by Myrberg [1963] and, in the case of the logistic map, by [May, 1976 ; Collet \& Eckmann, 1980; Feigenbaum, 1978], because it is linked to the presence of a discontinuity rather than to the passing through the value -1 of the eigenvalue. However, the same "boxes in boxes" structure [Gumowski \& Mira, 1980] is displayed. The figure 5b illustrates this phenomenon. It is even possible to determine the convergence rate [Feigenbaum, 1978] along the main diagonal, expressed as :

$$
\delta_{i}=\frac{\left(\lambda^{(2(i+1))-\lambda(2 i)}\right)}{\left(\lambda^{(2(i+1))}-\lambda^{(2(i+2))}\right)}
$$

where $\lambda^{(n)}$ stands for the parameter value where the period $n$ cycle appears. In this case, $\delta_{i}$ is found to converge to $4,699 \ldots$, that is, exactly the Feigenbaum constant determined on the logistic map case. The value of the accumulation point $\lambda^{(\infty)}$ is equal to the value found for the logistic map too. This is a striking fact, as the sawtooth map does not have the same number of critical points as the logistic map, and not the same bifurcation mechanism. Indeed, the only common point between the two maps is the use of quadratic expressions.

One finds smaller fishes not only between the two tails of a bigger fish, but also at the crossing of tails of two different fishes, which may have different degrees of complexity. In this case, it has been verified that the period of the smaller fish is the sum of the periods of the bigger ones, which leads to the expression of a second rule, illustrated in Fig. 5c: 


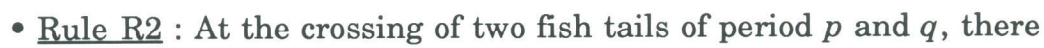
lies a fish of period $(p+q)$.

Because of the first rule, there is not only one fish at the crossing of two tails, but a whole "boxes in files" structure, produced by direct and inverse cascades.

Focusing attention on itineraries, it can be seen that the obtained sequence is not a simple concatenation of the starting sequences : indeed, the last term of the first itinerary and the first term of the second itinerary are inverted, $i$. e. transformed in $\mathrm{R}$ if it is $\mathrm{L}$ and $\mathrm{L}$ if it is $\mathrm{R}$. For example, the fish that lies below the intersection of the tails of $(R L)^{\infty}$ and $(\text { RLL })^{\infty}$ has periodic itinerary $(\text { RRLLL })^{\infty}$. This may again be expressed with

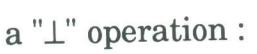

$$
(\mathrm{RL})_{\infty} \perp(\mathrm{RLL})_{\infty}=(\mathrm{RRLLL})_{\infty}
$$

and translated again into other notations as :

$$
\begin{aligned}
& \mathbf{h k} \perp \mathbf{h k k}=\mathbf{h} \mathbf{h k k} \mathbf{k} \\
& (2) \perp(3)=(14) \\
& 4 \mathrm{H} \perp 6 \mathrm{H}=15 \mathrm{~T}
\end{aligned}
$$

(note that the $15 \mathrm{~T}$ sequence has never been mentioned in studies on $\mathrm{SiC}$ polytypism).

This operation is not commutative : to obtain the appropriate result, one has to describe the itineraries by placing first the largest number possible of $R$, and set as a first operand the itinerary of the fish closest to the first diagonal ; this is for the case where the intersection lies in the part where there are more R's than L's in the itineraries, and the symmetrical procedure is made in the converse case. 
The series of itineraries for the period-doubling cascade arising from the hk fish is : hk, hhkk, hhkhkhkk, ..., a series for which Myrberg's ordering law [Myrberg, 1963 ; Metropolis et al... 1973 ; Mira, 1984], does not hold. It is an illustration of the fundamental difference between this cascade and the period-doubling cascade of the logistic map.

The infinite application of rules $\mathrm{R} 1$ and R2 leads to the fractal appearance of the diagram, that is, its self-similarity [Mandelbrot, 1989]. A close-up of Fig. 3b is shown in Fig. 3c, illustrating this fact.

The two-dimensional point of view permits to understand better the structure of the routes to chaos observed in one-dimensional families. Indeed, these are obtained by following any curve inside the $\left(\lambda_{1}, \lambda_{2}\right)$ plane. A striking fact is that the 2-dimensional regularity is lost when the curve is not a particular one : sudden interruptions appear, giving rise to irregular cascades where the rules $\mathrm{R} 1$ and $\mathrm{R} 2$ appear to be hidden.

\subsubsection{Coexistence of stable periodic regimes.}

It is easy to see that, in Fig. 3c, the intersection of two fish tails of different periods $p$ and $q$ has itself a complex structure where the period- $q$ points form a fractal ensemble inside the crossing area. This is actually due to the fact that the same initial value is taken for all the calculations, in a region where two periodic cycles are stable together, possibly with intertwined fractal-shaped attraction basins, as shown in [Grebogi et al..., 1987] for some two-humped 1D endomorphisms. A consequence of this fact is that all the fish tails, in contrast with the bodies, have a fractal structure. 


\subsubsection{Characterization of chaotic behavior.}

The algorithm described above is not sufficient to settle any evidence of chaos, so another one has been used, based on the criterion of sensitivity to initial conditions [Guckenheimer, 1979], expressed by the positiveness of the largest Lyapunov exponent, given by the following formula [Collet \& Eckmann, 1980] :

$$
\mu_{1}=\lim _{n \rightarrow \infty} \frac{1}{n} \sum_{t=0}^{n-1} \ln \left|\frac{d f(x)}{d x}\right|_{x=x_{t}}
$$

Accordingly, a computation of $\mu_{1}$ has been made for each point of the discretized $\left(\lambda_{1}, \lambda_{2}\right)$ plane, and a black pixel has been associated to the points where $\mu_{1}$ is positive. The result is shown in Fig. 6. The dark zone satisfying the chaoticity criterion is very similar to the zone of period $>156$ in Fig. $3 \mathrm{~b}$, to the extent of computer precision. It exhibits a fractal contour whose dimension can be measured, for example following the approach of Richardson \& Kaye [1989]. In this case, the value is found to be \#1.63, i.e., to the computation's precision, the ratio $\ln (4) / \ln (3)$, exactly as for von Koch's snowflake [Mandelbrot, 1989]. Indeed, Mira [1988] has already pointed out that the construction of the boxes-in-files structure is in some sense a "geometrical inverse" of Von Koch's construction.

\section{Symbolic Dynamics and Kneading Theory.}

In order to deal with the combinatorial properties of the sawtooth maps regardless to any particular analytic expression, attention will be paid to the itineraries associated to trajectories, and more precisely to those of $x_{-}^{*}=\lim _{x \rightarrow x^{*}, x<x^{*}} f(x)$ and $x_{+}^{*}=\lim _{x \rightarrow x^{*}, x>x^{*}} f(x)$, called kneading sequences [Milnor \& Thurston, 1988]. It will be seen that they are of particular interest in the characterization of the various cascades and in the proof of chaos. Some definitions are needed prior to any further study. 


\subsection{Admissible sequences.}

A simple question is : does any sequence of R's, L's, and C's represent an itinerary of a sawtooth map? In other words, what are the criterions for a sequence to be admissible as an itinerary? Two cases may occur :

- the sequence is made of an infinite suite of R's and L's only ;

- the sequence includes the letter $\mathrm{C}$ at a given index.

In the first case, there is no particular restriction in the choice of letter, while in the second case, what follows the letter $\mathrm{C}$ is the itinerary of either $x_{+}^{*}$ or $x_{-}^{*}$, according to the particular definition of the function. For this reason, it is preferred to "cut" the sequence after the letter $\mathrm{C}$, which leads to the following admissibility criterion [Collet \& Eckmann, 1980 ; Metropolis et al., 1973] :

- A sequence is admissible as an itinerary if and only if it is constituted of either an infinite sequence of $R$ and $L$, or of a sequence of $R$ and $\mathrm{L}$ followed by the letter $\mathrm{C}$.

\subsection{Numerical representation of itineraries.}

It can be seen to which extent this definition suits the case of sawtooth maps by establishing a 1-1 correspondence between itineraries and real numbers of the unit interval, inspired from [Isola \& Politi, 1990] :

- Given an admissible sequence $\mathrm{I}=\mathrm{U}_{0} \mathrm{U}_{1} \mathrm{U}_{2} \mathrm{U}_{3} \ldots$, the real number $r$ associated to $I$ is the following, in binary notation :

$$
r=T(\mathrm{I})=0 . d_{0} d_{1} d_{2} d_{3} \ldots d_{i} \ldots \text { with }\left\{\begin{array}{l}
d_{\mathrm{i}}=0 \text { if } \mathrm{U}_{\mathrm{i}}=\mathrm{L} \\
d_{i}=1 \text { if } \mathrm{U}_{\mathrm{i}}=\mathrm{R} \text { or } \mathrm{C}
\end{array}\right.
$$


Thus, sequence $\mathrm{C}$ corresponds to 0.1 in binary notation or $1 / 2$ or 0.5 in common representations ; LC corresponds to 0.01 or $1 / 4$ or 0.25 ; RC corresponds to 0.11 or $1 / 2+1 / 4$ or 0.75 , etc... $T$ is a 1 -to- 1 relation, whose reciprocal transformation is defined as follows :

- Let $r$ be a real number of $[0,1]$ such that its decimal part is $0 . d_{0} d_{1} d_{2} d_{3} \ldots d_{n-1} 1000 \ldots$. in binary notation. Then the associated itinerary is :

$$
\mathrm{I}=S(r)=\mathrm{U}_{0} \mathrm{U}_{1} \mathrm{U}_{2} \mathrm{U}_{3} \ldots \mathrm{U}_{n-1} \mathrm{C} \text { with }\left\{\begin{array}{l}
\mathrm{U}_{\mathrm{i}}=\mathrm{L} \text { if } d_{i}=0 \\
\mathrm{U}_{\mathrm{i}}=\mathrm{R} \text { if } d_{i}=1
\end{array}\right.
$$

If the decimal part is infinite, the letter $\mathrm{C}$ is never encountered. Note that the number 1 can be equivalently represented as $0.111111 \ldots$ and has associated itinerary $\mathrm{R}^{\infty}$.

\subsection{The shift operator.}

If $\mathrm{I}=\mathrm{U}_{0} \mathrm{U}_{1} \mathrm{U}_{2} \mathrm{U}_{3} \ldots$ is the itinerary associated to the real $x$, then the itinerary associated to $f(x)$ is $\mathrm{I}^{\prime}=\mathrm{U}_{1} \mathrm{U}_{2} \mathrm{U}_{3} \mathrm{U}_{4} \ldots=\sigma(\mathrm{I})$. The operation $\sigma$ is simply a rightwards shift operation.

Let us examine how this operation is translated in terms of binary representation of numbers, by comparing $r=0 . d_{0} d_{1} d_{2} d_{3} \ldots$ and $T(\sigma(S(r)))=0 . d_{1} d_{2} d_{3} d_{4} \ldots$. Two cases can occur :

-If $\mathrm{r}<0.1$, then it is written $0.0 d_{1} d_{2} d_{3} \ldots$. The effect of $\sigma$ is to move from :

$$
\left(0+0 \times 1 / 2+d_{1} \times 1 / 4+d_{2} \times 1 / 8+\ldots\right)
$$

to :

$$
\left(0+d_{1} \times 1 / 2+d_{2} \times 1 / 4+\ldots\right)
$$

which is nothing else than multiply $r$ by 2 . 
-If $\mathrm{r} \geq 0.1$, then it is written $0.1 d_{1} d_{2} d_{3} \ldots$. The effect of $\sigma$ is to move from :

$$
\left(0+1 \times 1 / 2+d_{1} \times 1 / 4+d_{2} \times 1 / 8+\ldots\right)
$$

to :

$$
\left(0+d_{1} \times 1 / 2+d_{2} \times 1 / 4+\ldots .\right)
$$

which is the same thing as remove $1 / 2$ to $r$, and multiply the result by 2 .

Finally, the equivalent of $\sigma$ for real numbers is the following function :

$$
\begin{aligned}
\mathrm{g}:[0 ; 1] & \rightarrow[0 ; 1] \\
r & \mapsto\left\{\begin{array}{c}
2 r \text { si } r<1 / 2 \\
2(r-1 / 2) \text { si } r \geq 1 / 2
\end{array}\right.
\end{aligned}
$$

The scheme below shows the relations between $\sigma$ and $g$ :

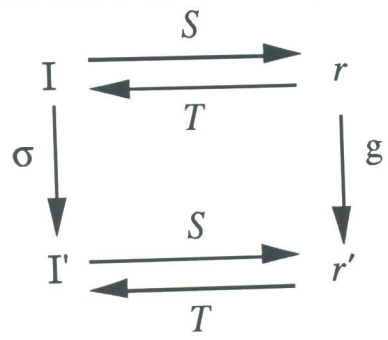

The function $\mathrm{g}=T \circ \sigma \circ S$ is the simplest possible sawtooth map, whose graph is made of two line segments, as shown in Fig. 7. It is related to the functions defined in (1) by a transformation which is continuous, derivable and increasing on $[0,1 / 2[$ and on $] 1 / 2,1]$ - a fact that does not ensure topological conjugacy with these functions. 


\subsection{A natural ordering of itineraries.}

The letters $\mathrm{L}, \mathrm{C}$ and $\mathrm{R}$ are related to values of $x$ respectively inferior, equal and superior to $x^{*}$. As a consequence, it is natural to introduce an ordering between these letters :

$$
\mathrm{L}<\mathrm{C}<\mathrm{R}
$$

In a more general way, an ordering can be applied to sequences of any length, based on the fact that $f$ is increasing everywhere it is continuous :

- If $\mathrm{I}=\mathrm{U}_{0} \mathrm{U}_{1} \mathrm{U}_{2} \mathrm{U}_{3} \ldots$ and $\mathrm{J}=\mathrm{V}_{0} \mathrm{~V}_{1} \mathrm{~V}_{2} \mathrm{~V}_{3} \ldots$ are two sequences and $i$ the first index for which $\mathrm{U}_{i} \neq \mathrm{V}_{i}$, then $\mathrm{I}>\mathrm{J} \Leftrightarrow \mathrm{U}_{i}>\mathrm{V}_{i}$.

This relation is simpler than the one defined for itineraries of the logistic mapping, as this function is decreasing past $x^{*}$.

A useful remark has to be made then : as there exists a 1-1 relation between itineraries and real numbers of $[0,1]$, the ordering defined above is directly a transposition of the usual ordering of real numbers, so that :

- If I and $\mathrm{J}$ are two itineraries, then $\mathrm{I}<\mathrm{J} \Leftrightarrow T(\mathrm{I})<T(\mathrm{~J})$.

\subsection{The kneading sequences.}

Two itineraries are particularly interesting for a given function $f$ : the itinerary of $f\left(x_{-}^{*}\right)$, with $x_{-}^{*}=\lim _{x \rightarrow x^{*}, x<x^{*}} f(x)$, hereafter denoted by $\mathrm{K}_{-}$, and the one of $f\left(x_{+}^{*}\right)$, with $x_{+}^{*}=\lim _{x \rightarrow x^{*}, x>x^{*}} f(x)$, denoted by $K_{+}$, and called kneading sequences because they represent in some sense a maximum and a minimum for the possible sequences [Milnor \& Thurston, 1988]. In the case of the functions $f$, it is evident that, when $\left(\lambda_{1}, \lambda_{2}\right) \in[0,5,1] \times[0,5,1], \mathrm{K}$. begins with an $\mathrm{R}$ and $\mathrm{K}_{+}$begins with an $\mathrm{L}$. 
The following developments are essentially inspired from the works of [Zheng \& Lu, 1991] concerning the gap map [De Souza Vieira, et $a l .$, 1987, Chia \& Tan, 1992]. The main idea is that a given endomorphism is characterized by its kneading sequences. Indeed, the "kneading character" leads to a condition for any itinerary I to be admissible for the map, written in terms of $\mathrm{K}_{+}$and $\mathrm{K}_{-}$:

- $\boldsymbol{L}(\mathrm{I}) \leq \mathrm{K}$. and $\boldsymbol{R}(\mathrm{I}) \geq \mathrm{K}_{+}$, where $\boldsymbol{L}(\mathrm{I})=\{\mathrm{Y} \mid \mathrm{I}=\mathrm{XLY}\}$

$$
\text { and } \boldsymbol{R}(\mathrm{I})=\{\mathrm{Y} \mid \mathrm{I}=\mathrm{XRY}\}
$$

$\boldsymbol{L}(\mathrm{I})$ is the set of subsequences of I that follow the letter $\mathrm{L}$, and $\boldsymbol{R}(\mathrm{I})$ is similarly defined.

\subsection{The skeleton of the bifurcation diagram.}

Applying criterion (16) to $K_{+}$and $K_{-}$themselves, it is possible to determine systematically which couples $\left(\mathrm{K}_{-}, \mathrm{K}_{+}\right)$do actually represent a sawtooth map. Indeed, they have to knead themselves and each other, which can be translated into the following conditions :

$$
\boldsymbol{L}\left(\mathrm{K}_{-}, \mathrm{K}_{+}\right)<\mathrm{K}_{-} \text {and } \boldsymbol{R}\left(\mathrm{K}_{-}, \mathrm{K}_{+}\right)>\mathrm{K}_{+}
$$

In particular, (17) implies that $\mathrm{K}_{+}$has to be minimal, i.e. inferior to any of its shifts, and that $K_{\text {. }}$ has to be maximal.

Focusing the interest on finite (superstable) kneading sequences, which are in some sense boundaries for regions of periodic behavior, a skeleton is drawn in Fig. 8, where lines in a $\left(K_{,}, K_{+}\right)$plane stand for allowed sequences of length up to 6 . Any crossings between these lines satisfy the conditions (17); the contact points satisfy only $L\left(\mathrm{~K}_{+}\right) \leq \mathrm{K}_{\text {- }}$ and $\boldsymbol{R}\left(\mathrm{K}_{-}\right) \geq \mathrm{K}_{+}$. These contacts do represent maps if the following additional condition is satisfied : 
- Let $\left(\mathrm{K}_{-}, \mathrm{K}_{+}\right)$be equal to (XC,YC), with $\boldsymbol{L}(\mathrm{YC}) \leq \mathrm{XC}$ and $\boldsymbol{R}(\mathrm{XC})$ $\geq$ YC. This couple represents a map if and only if $\boldsymbol{R}\left((\mathrm{XL})^{\infty}\right)>\mathrm{YC}$ or $\mathrm{L}\left((\mathrm{YR})^{\infty}\right)$ $<\mathrm{XC}$.

The diagram in Fig. 8 displays a striking similarity with Fig. $3 \mathrm{~b}$, which confirms the interest of the kneading theory in the understanding of the bifurcations of the sawtooth map. As an example, the limiting curves of the body of the period-2 fish correspond to the collision of one of the two points of the periodic orbit (of sequence $(\mathrm{LR})^{\infty}$ ) with the discontinuity, i. e., where :

$$
\begin{aligned}
\mathrm{K} . & =\mathrm{RC}, \\
\text { or } \quad \mathrm{K}_{+} & =\mathrm{LC} .
\end{aligned}
$$

(18a) and (18b) are the exact counterparts of Eqs. (7a) and (7b) in the $\left(\mathrm{K}_{-}, \mathrm{K}_{+}\right)$plane. Keeping in mind this relationship between the skeleton and the bifurcation diagram of the family (1), the properties of the former will be exploited to have a more precise insight of the latter.

\subsection{Generation of contacts and crossings in the skeleton.}

The structure of the skeleton is owed essentially to a main property, described in the following fundamental theorem :

- $\underline{\text { Theorem T1 }}$ : If $\left(\mathrm{K}_{,}, \mathrm{K}_{+}\right)=(\mathrm{XC}, \mathrm{YC})$ is a contact, then $(\mathrm{XRYC}, \mathrm{YC})$ and (XC, YLXC) are also contacts.

The proof, as inspired from [Zheng \& Lu, 1991], is reported to Appendix A.1. An immediate consequence of $\mathrm{T} 1$ is that a third contact - in fact, a crossing - is generated : (XRYC, YLXC). The four couples enclose a rectangle $[\mathrm{XC}, \mathrm{XRYC}] \times[\mathrm{YLXC}, \mathrm{YC}]$, hereafter referred to as the direct shadow of the crossing (XRYC,YLXC). The area defined by $\mathrm{K}<\mathrm{XRYC}$ and 
$\mathrm{K}_{+}>$YLXC, which contains the direct shadow, will be merely called the shadow of (XRYC,YLXC) [Zheng \& Lu, 1991]. Inside a direct shadow, there is no other contact between finite sequences, as proved in Appendix A.2. From this proof, it is also seen that the only possible couple of kneading sequences inside this area is $\left((\mathrm{XRYL})^{\infty},(\mathrm{YLXR})^{\infty}\right)$. Even though, there are normally many possible itineraries for the latter map, because any itinerary admissible for a contact belonging to the shadow of a crossing is also admissible for that top crossing. It should be kept in mind that all these itineraries can correspond to stable or unstable orbits for a particular form of the mapping. Determining which periodic orbits are stable and which are not is beyond the scope of this paper. However, it can be pointed out, as the direct shadows are the counterparts of the fish bodies in Fig. 3b, that the only stable periodic orbit inside a fish body corresponding to the direct shadow of (XRYC,YLXC) appears to be (XRYL) $)^{\infty}$.

On the basis of this last remark, the skeleton of Fig. 8 has been colored in the same way as the bifurcation diagram of Fig. 3b, allowing to enhance further the similarity between them. The zones equivalent to the fish tails appear then as the strips enclosed between the line corresponding to the curve limiting the body and the inside of the tails, and the accumulation locus of the lines corresponding to the cascade closest to the tail. For example, the period- 2 fish is represented by the direct shadow of (RC,LC) for its body, by the lines $\mathrm{K}=\mathrm{RC}$ and $\mathrm{K}_{+}=\mathrm{LC}$ for the inside limit of its tails, and by the accumulation loci of the families of lines $\mathrm{K}_{-}=(\mathrm{RL})^{\mathrm{n}} \mathrm{C}$ and $\mathrm{K}_{+}=(\mathrm{LR})^{\mathrm{n}} \mathrm{C}$. Indeed, it is easy to prove that $(\mathrm{RL})^{\infty}$ is admissible if $\mathrm{K} \leq$ $\mathrm{RC}$ or $\mathrm{K}_{+} \geq \mathrm{LC}$; however, (i) this itinerary coexists only with $\mathrm{R}^{\infty}$ and $\mathrm{L}^{\infty}-$ which are unstable - inside the direct shadow of (RC,LC), and (ii) it coexists with many other periodic orbits - possibly stable too - outside this region. This latter fact will be used in the demonstration of topological chaos. 
Another consequence of theorem $\mathrm{T} 1$ is an explanation of the rules $\mathrm{R} 1$ and $\mathrm{R} 2$ in the bifurcation diagram. The generation of a direct shadow by $\mathrm{T} 1$ out of a crossing implies the character of additivity of rules $\mathrm{R} 1$ and R2. Namely, if $l(\mathrm{X})$ is the length of $\mathrm{X}$ and $l(\mathrm{Y})$ the length of $\mathrm{Y}$, then $l(\mathrm{XRYC})=l(\mathrm{YLXC})=l(\mathrm{XC})+l(\mathrm{YC})=l(\mathrm{X})+l(\mathrm{Y})+2$. If $(\mathrm{XC}, \mathrm{YC})$ is a contact, then $\mathrm{T} 1$ leads to rule $\mathrm{R} 1$, and if it is a crossing, then it corresponds to rule R2. This follows from the fact that if $(\mathrm{XC}, \mathrm{YC})$ is a contact, then it is adjacent to some direct shadow, while if it is a crossing, it is not adjacent to any direct shadow and consequently represents the intersection of two tails.

\subsection{Self-similarity of the diagram.}

The repetition ad infinitum of theorem $\mathrm{T} 1$ (or of rules $\mathrm{R} 1$ and R2) leads to the self-similar structure of the skeleton, and of the bifurcation diagram related to it. Indeed, it is possible to recognize some transformations of the $\left(\mathrm{K}, \mathrm{K}_{+}\right)$plane which relate parts of the diagram with smaller ones ; they can be very useful for a study of the renormalizations associated to the bifurcations.

For instance, the direct and inverse cascade may be described in terms of the two following transformations :

$$
\begin{array}{r}
\mathcal{F}: \mathrm{R} \rightarrow \mathrm{RL} \\
\mathrm{L} \rightarrow \mathrm{L} \\
\mathrm{C} \rightarrow \mathrm{C} \\
\text { and } \mathrm{G}: \mathrm{L} \rightarrow \mathrm{LR} \\
\mathrm{R} \rightarrow \mathrm{R} \\
\mathrm{C} \rightarrow \mathrm{C}
\end{array}
$$

As in [Zheng \& Lu, 1991], it is easy to see that $\mathcal{F}$ and $\mathcal{G}$ are order preserving, and that, if $\left(K_{,}, K_{+}\right)$is a contact, then $\left(\mathcal{F}\left(K_{-}\right), L \mathcal{F}\left(K_{+}\right)\right)$and 
The Sawtooth Map and the growth of $\mathrm{SiC}$ polytypes

(Rg(K.), $\left.9\left(\mathrm{~K}_{+}\right)\right)$are contacts too. As a matter of fact, the band defined by $\mathrm{C} \leq$ $\mathrm{K} . \leq \mathrm{RC}$ and $\mathrm{K}_{+} \leq \mathrm{C}$ has an image by $\mathcal{F}$ in the band defined by $\mathrm{C} \leq \mathrm{K} \leq \mathrm{RLC}$ and $\mathrm{K}_{+} \leq \mathrm{LC}$. In particular, the direct shadow of (RC,LC) (body of the period-2 fish) is transformed into the direct shadow of (RLC,LLC) (body of one period-3 fish). The iterated action of (F,LF ) is displayed in Fig. 9. $(\mathrm{R} 9,9)$ acts in a symmetrical way. $\mathcal{F}$ and 9 are related to the building and ordering of Farey sequences.

The period doublings may be described in terms of the following transformation :

$$
\begin{gathered}
\mathscr{O}: \mathrm{R}->\mathrm{RL} \\
\mathrm{L}->\mathrm{LR} \\
\mathrm{C}->\mathrm{C} .
\end{gathered}
$$

$\mathscr{O}$ preserves the ordering, and it can be seen that if $\left(\mathrm{K}, \mathrm{K}_{+}\right)$is a

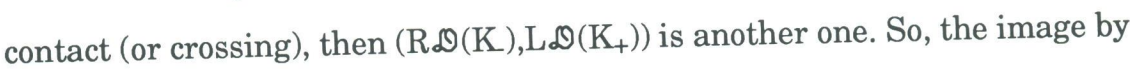
$(\mathrm{R} D, L \mathscr{Q})$ of $(\mathrm{RC}, \mathrm{LC})$ is $(\mathrm{RRLC}, \mathrm{LLRC})$ : this corresponds to the subharmonic cascade mentioned above.

The above three transformations do not allow to deduce all the parts of the diagram out of the equivalent of the period-2 fish. Indeed, the fishes generated by rule $\mathrm{R} 2$, with exception of the $p=q$ case, are forgotten. To be more complete, other more complicated transformations, but with similar properties, should be defined.

\subsection{The boundary of chaos.}

The skeleton is made of zones which are the direct shadows of crossings, and zones which are not. The first kind is directly related to fish bodies, and the second to tails. A glance in Figs. $3 \mathrm{~b}$ and $3 \mathrm{c}$ indicates that chaos is more especially linked to the structure of the tails. Accordingly, a demonstration on the onset of topological chaos will be made for a crossing 
The Sawtooth Map and the growth of SiC polytypes

which has no direct shadow. If (XC,YC) is a contact, then (XRYRYC, YLXC) is such a crossing - actually, a crossing between two fish tails. As proved in the appendix A.3, any itinerary $I=S_{1} S_{2} \ldots S_{n} \ldots$, where $S_{i}$ is either XRYL = $\mathrm{E}_{1} \mathrm{~L}$ or $\mathrm{XRYRYL}=\mathrm{E}_{2} \mathrm{~L}$ is admissible for this crossing. A consequence is that the number of periodic points of a given length $n$ has the following property :

$$
\mathrm{N}_{n} \geq \mathrm{N}_{n-l_{1}}+\mathrm{N}_{n-l_{2}}
$$

where $l_{1}$ and $l_{2}$ are the respective lengths of $\mathrm{E}_{1} \mathrm{~L}$ and $\mathrm{E}_{2} \mathrm{~L}$. This implies that $\mathrm{N}_{n} \geq 2 \mathrm{~N}_{n-l_{1}}$, and, going further, that $\mathrm{N}_{n} \geq 2^{n / l_{1}}$. The topological entropy [Grassberger \& Procaccia, 1984] is defined as :

$$
h(f)=\lim _{n \rightarrow \infty} \frac{\mathrm{N}_{n}}{n}
$$

In this case, $h(f)=\lim _{n \rightarrow \infty} \frac{N_{n}}{n} \geq \frac{1}{n} \log \left(2^{n / h_{1}}\right)$, or $h(f) \geq \frac{1}{l_{1}} \log (2)>0$. The positiveness of $h(f)$ ensures the presence of topological chaos. Thus, all points whose shadows contain a crossing between two fish tails exhibit topological chaos. Remember that it is only a prerequisite for "observable" chaos. As a matter of fact, Fig. 6 seems to show that the fish tails are not chaotic regions ; however, it is recalled that the algorithm used to generate it involves only one initial value, while the tails are possibly domains of coexistence of cycles. If the calculations were carried on many initial values, the chaotic domain would extend itself to the union of all the fish tails.

\section{Comparison with Experimental Data on Polytypism.}

Two main questions arise concerning the adequation of the model with the reality of polytypism, on the combinatorial level :

(i) Is the sawtooth map model able to describe all the known polytypes? 
(ii) Do the known polytypic sequences settle themselves according to some regularity inside the bifurcation diagram?

The first question has a positive answer since for any integer number $n$ all sequences of length $\leq n$ are produced by the model. In other words, the bifurcation diagram contains domains of stability for all imaginable periodic sequences - and when the period is of infinite length, these are aperiodic sequences. A demonstration is sketched below.

Let $(\mathrm{P})^{\infty}$ be a periodic itinerary of period $p$. There always exists an $m \leq p$ such as $\left(\sigma^{m}(\mathrm{P})\right)^{\infty}=(\mathrm{M})^{\infty}$ is maximal and an $n \leq p$ such as $\left(\sigma^{n}(\mathrm{P})\right)^{\infty}$ $=(\mathrm{Q})^{\infty}$ is minimal. Because of the periodicity of the sequences, we have $\boldsymbol{L}(\mathrm{Q})=\boldsymbol{L}(\mathrm{M}) \leq \mathrm{M}$ and $\boldsymbol{R}(\mathrm{Q})=\boldsymbol{R}(\mathrm{M}) \geq \mathrm{Q}$. Consequently, $\left((\mathrm{M})^{\infty},(\mathrm{Q})^{\infty}\right)=\left(\mathrm{K}_{1}, \mathrm{~K}_{2}\right)$ is a couple of kneading sequences for which $(\mathrm{P})^{\infty}$ is admissible.

$(\mathrm{M})^{\infty}$ and $(\mathrm{Q})^{\infty}$ differ only by a certain number of shifts, $i$. $e$. $\sigma^{(m-n) \bmod p(\mathrm{Q})}=\mathrm{M}$. Suppose that $\mathrm{m} \leq \mathrm{n}$ (the converse case is similar) and call $\mathrm{U}$ the sequence $\mathrm{P}_{m} \mathrm{P}_{m+1} \ldots \mathrm{P}_{n-1}$. and $\mathrm{V}$ the sequence $\mathrm{P}_{n} \mathrm{P}_{n+1} \ldots \mathrm{P}_{p} \mathrm{P}_{1} \mathrm{P}_{2} \ldots \mathrm{P}_{m-1}$. Then $\mathrm{K}_{1}=(\mathrm{UV})^{\infty}$ and $\mathrm{K}_{2}=(\mathrm{VU})^{\infty}$. There remains to show that $\mathrm{U}=\mathrm{XR}$ and $\mathrm{V}=\mathrm{YL}$, where $\mathrm{XC}$ et $\mathrm{YC}$ are respectively maximal and minimal and are in contact.

If $(\mathrm{UV})^{\infty}$ is maximal and non-trivial, then it begins with an $\mathrm{R}$. In this case, $\mathrm{V}$ ends up with an $\mathrm{L}$, because otherwise $\sigma^{p-1\left((\mathrm{UV})^{\infty}\right)}$ would begin with an extra $R$ and would be superior to (UV) ${ }^{\infty}$, contradicting its maximality. The same demonstration is used to prove that $U$ ends with an $R$. So, in general, there are always an $X$ and a $Y$ so that $U=X R$ and $V=Y L$. $\left(\mathrm{K}_{1}, \mathrm{~K}_{2}\right)$ can be written $\left((\mathrm{XRYL})^{\infty},(\mathrm{YLXR})^{\infty}\right)$. Furthermore, $\boldsymbol{L}\left((\mathrm{XRYL})^{\infty}\right) \leq$ $(\mathrm{XRYL})^{\infty}$ implies that $\boldsymbol{L}(\mathrm{XC}) \leq \mathrm{XC}$ (again, the opposite would lead to contradict the maximality of $\left.(\mathrm{XRYL})^{\infty}\right)$. This is enough to prove that $\mathrm{XC}$ is maximal ; similarly, one proves that $\mathrm{YC}$ is minimal, that $\boldsymbol{R}(\mathrm{XC}) \geq \mathrm{YC}$, and 
The Sawtooth Map and the growth of SiC polytypes

that $\boldsymbol{L}(\mathrm{YC}) \leq \mathrm{XC}$. Theorem T1 may then be applied to generate the direct shadow of (XRYC,YLXC), within which $(\mathrm{P})^{\infty}$ is admissible. This shadow is directly related to the body of the period-p fish exhibiting sequence $(\mathrm{P})^{\infty}$. This suggests a systematic method to locate the direct shadow and the fish body associated to any periodic polytype, as a tool to answer the second question.

Let $\mathrm{I}=(\mathrm{P})^{\infty}$. Determine the maximal and minimal shifted sequences :

$$
(\mathrm{M})^{\infty}=\max _{0<i<p} \sigma^{i}(\mathrm{I}) \text { and }(\mathrm{Q})^{\infty}=\min _{0<i<p} \sigma^{i}(\mathrm{I}) .
$$

Replacing the last letter of $\mathrm{M}$ and $\mathrm{Q}$ by $\mathrm{C}$ gives the co-ordinates of the crossing (XRYC,YLXC). The numerical translation $T$ helps to locate them accurately.

This method is easily transposable for the "fish diagram", because a direct shadow has a counterpart in a fish body. The equations of the curves limiting the body of the $(\mathrm{P})^{\infty}$ fish are :

$$
\begin{aligned}
& f_{\mathrm{M}_{p-1}} \circ f_{\mathrm{M}_{p-2}} \circ \ldots \circ f_{\mathrm{M}_{1}} \circ f_{\mathrm{L}}(1 / 2)=1 / 2, \\
\text { and } \quad & f_{\mathrm{Q}_{p-1}} \circ f_{\mathrm{Q}_{p-2}} \circ \ldots \circ f_{\mathrm{Q}_{1}} \circ f_{\mathrm{R}}\left(1 / 2^{+}\right)=1 / 2,
\end{aligned}
$$

where $p$ is the length of $\mathrm{M}$ and $\mathrm{Q}$, and $f_{\mathrm{S}}(x)=4 \lambda_{1} x(1-x)$ if $\mathrm{S}=\mathrm{L}$ and $f_{S}(x)=1-\left[4 \lambda_{2} x(1-x)\right]$ if $S=R$.

Figure 10 is the result of a program designed to display in color the only fishes that correspond to all the known polytypes of $\mathrm{SiC}$ with period $\leq 50$, as listed in [Gmelin Handbook, 1989]. The majority of the polytypes lie in the part where $\lambda_{2} \geq \lambda_{1}$ : this is due to the fact that most of them include more $\mathbf{k}$ layers than $\mathbf{h}$ ones. There is no real contiguity between different polytypes in the diagram. In the part generated by direct 
and inverse cascades, there appears approximately only one every two polytypes. Actually, most of them are made of "basic sequences" like (22), (23), (33), etc... in Zhdanov notation [Zhdanov, 1945], while the sawtooth map cascades involve simpler basic sequences like (2), (3), (4), etc.... Finally, some polytypes are represented by very small fishes. It is to be pointed out that, as the precise connection of $\lambda_{1}$ and $\lambda_{2}$ with physical parameters such as pressure and temperature is not known, the diagram of Fig. 3a is not intended to account of the relative abundance of the known polytypes. Simple changes in parameter dependencies in (1) may dramatically change the shape and relative size of the fishes. However, the most frequent polytypes $(\mathbf{k})^{\infty},(\mathbf{h k k})^{\infty},(\mathbf{h k h k k})^{\infty}$, and $(\mathbf{h k})^{\infty}$ correspond to large areas in the diagram.

\section{Conclusion.}

A dynamical model based on the iteration of sawtooth maps has been proposed to describe the physical phenomenon of polytypism in CVD/CVI SiC growth. As it involves only one dynamical variable, it is believed to be a very simple approximation to the physical reality; however, it is enough to account for the growth of any periodic or chaotic sequence. Further applicability of this model relies on more accurate determination of layer deformation in polytypes of $\mathrm{SiC}$ or other materials [Verma \& Krishna, 1966 ; Baronnet, 1980].

The sawtooth map has also an interest from a more theoretical point of view, as it has a very simple underlying kneading theory. The main result is that all routes to chaos seem to derive from the repeated application of theorem T1; the two-dimensional representation has helped to a clearer understanding of these routes. Maybe is it an appropriate tool for studies in renormalization theory and other topics in dynamics of discrete systems. 
The Sawtooth Map and the growth of SiC polytypes

\section{Acknowledgements}

This work has been supported by both CNRS and SEP through grants to the author. 


\section{References.}

BARONNET, A. [1980] "Polytypism in micas : a survey with emphasis on the crystal growth aspect", Current Topics in Materials Science, 5, E. Kaldis ed. (North-Holland, Amsterdam).

CHENG, C., HEINE, V. \& NEEDS, R. J. [1990] "Atomic relaxation in silicon carbide polytypes", J. Phys.: Condensed Matter 2, 5115-5134.

CHIA, T. T. \& TAN, B. L. [1992] "Results for the discontinuous logistic map", Phys. Rev. A , 45, 8441-8449.

COLLET, P. \& ECKMANN, J. P. [1980] "Iterated maps on the interval as dynamical systems", Progress in Physics, 1, Jaffe, A. \& Ruelle, D. eds. (Birkhäuser, Basel-Boston-Stuttgart)

DE SOUZA VIEIRA, M. C., LAZO, E. \& TSALLIS, C. [1987] "New road to chaos", Phys. Rev. A, 35, 945-948.

FEELY, O. \& CHUA, L. O. [1992] "Nonlinear dynamics of a class of analogto-digital converters", Int. J. of Bifurcation and Chaos, 2, 325-340.

FEIGENBAUM, M. J. [1978] "Quantitative universality for a class of nonlinear transformations", J. Stat. Phys., 19, 25-52.

FISHER, G. R. \& BARNES, P. [1990] "Towards a unified view of polytypism in silicon carbide", Phil. Mag., 61, 217-236.

FOURNIER-PRUNARET, D. [1991] "The bifurcation structure of a family of degree one circle endomorphisms", Int. J. of Bifurcation and Chaos, 1, 828-838.

Gmelin Handbook of Chemistry, Si suppl., vol B2 [1989]. 
GRASSBERGER, P. \& PROCACCIA, I. [1984] "Measuring the strangeness of strange attractors",Physica, 13D, 34 .

GREBOGI, C., KOSTELICH, E., OTT, E. \& YORKE, J. A. [1987] "Multidimensioned intertwined basin boundaries : basin structure of the kicked double rotor", Physica 25D, 347-360.

GUCKENHEIMER, J. [1979] "Sensitive dependence on initial conditions for one-dimensional maps", Comm. Math. Phys., 70, 133-160.

GUMOWSKI, I. \& MIRA, C. [1980] Dynamique Chaotique, ed. Cepadues, Toulouse.

ISOLA, S. \& POLITI, A. [1990] "Universal encoding for unimodal maps", J. Stat. Phys., 61, 263-291.

JAGODZINSKI, H. [1949], "One-dimensional Disorder in Crystals and its influence on X-Ray Diffraction", Acta Cryst., 2, 201-207.

KAYE, B. H. [1989] "A random walk through fractal dimensions", 1st ed. (VCH Verlagsgesellschaft, Weinheim, FRG - New York).

LEONOV, N. N. [1959] "Transformations d'une droite en elle-même", Radiofisica, 2, 942 .

LEONOV, N. N. [1960] "Transformation ponctuelle d'une droite en ellemême, discontinue, linéaire par morceaux", Radiofisica, 3, 496.

LEONOV, N. N. [1960] "Théorie des transformations discontinues d'une droite en elle-même", Radiofisica, 3, 872.

MANDELBROT, B. [1989] "Les objets fractals", 3rd ed. (Flammarion, Paris)

MAY, R. M. [1976] "Simple mathematical models with very complicated dynamics", Nature, 261, 459-467. 
METROPOLIS, M., STEIN, M. L. \& STEIN, P. R. [1973] "On finite limit sets for transformations on the unit interval", J. Comb. Theory (A), 15, 25-44.

MILNOR, J. \& THURSTON, W. [1988] in "Dynamical Systems", Lect. Notes in Math. $\mathbf{N}^{\circ}$ 1342, ed. Alexander, J. C. (Springer Verlag, Berlin).

MIRA, C. [1978], "Sur la structure des bifurcations des difféomorphismes du cercle", C. R. A. S A $287,883$.

MIRA, C. [1984], "Rotation sequences and bifurcations structure of onedimensional endomorphisms", Int. Symp. on Iteration Theory and its Functional Equations, Vorarlberg (Austria).

MIRA, C. [1988] "Nombres de rotation algébriques quadratiques et suites de rotation de Myrberg correspondantes", C. R. A. S. series I, 307, 803806.

MYRBERG, P. J. [1963] , "Iteration der reellen Polynome zweiten Graden", Ann. Acad. Sci. Fenn., series A, 336, 1-10.

RAMSDELL, L. S. [1947] "Studies on silicon carbide", Am. Mineralogist, 32, 64-82.

SHARKOVSKII, A. N. [1964] "Coexistence of cycles for a continous map of a segment into itself", Ukr. Mat.Zh., 16 (1964), 61-70 ;

STEFAN, P. [1977] "A theorem of Sharkovskii on the existence of periodic orbits of continuous endomorphisms of the real line", Comm. Math. Phys., 54, 237-248.

VERMA, A. A. \& KRISHNA, P. [1966] Polymorphism and polytypism in crystals (Wiley \& Sons, New York). 
VIGNOLES, G. L. [1992] "Atomic relaxation and dynamical generation of ordered and disordered chemical vapour infiltration (CVI) $\mathrm{SiC}$ polytypes", J. Crystal Growth 118, 430-438.

ZHDANOV, G. S. [1945] "The numerical symbol of compact stackings of spheres and its applications on the theory of compact stackings", C. $R$. Acad. Sci. URSS, 48, 43.

ZHENG, W.-M. \& LU, L.-S. [1991], "Boundary of chaos for the gap map", Comm. Theor. Phys., 15, 161-168. 


\section{Appendices.}

\section{A. 1. Demonstration of theorem T1.}

Let it be proved for $\left(\mathrm{K}_{-}^{\prime}, \mathrm{K}_{+}\right)=(\mathrm{XRYC}, \mathrm{YC})$ only : The other case is analogous. The following conditions have to be fulfilled by the couple :

$$
\begin{aligned}
& \mathrm{K}_{+} \text {is minimal, i. e. : } \mathrm{K}_{+}<\sigma^{i}\left(\mathrm{~K}_{+}\right), i>0 ; \\
& \mathrm{K}_{-}^{\prime} \text { is maximal, i. e. : } \mathrm{K}_{-}^{\prime}>\sigma^{i}\left(\mathrm{~K}_{-}^{\prime}\right), i>0 ; \\
& \boldsymbol{L}\left(\mathrm{K}_{+}\right) \leq \mathrm{K}^{\prime} \text {. and } \boldsymbol{R}\left(\mathrm{K}_{-}^{\prime}\right) \geq \mathrm{K}_{+} .
\end{aligned}
$$

The first condition is realized by hypothesis. $L\left(K_{+}\right) \leq K^{\prime}$ is true since $K^{\prime}>K_{-}$(that is, $\mathrm{XRYC}>\mathrm{XC}$ ). It remains to show that $\boldsymbol{L}\left(\mathrm{K}^{\prime}{ }_{-}\right)<\mathrm{K}^{\prime}$, which implies the maximality of $\mathrm{K}_{-}^{\prime}$, and that $\boldsymbol{R}\left(\mathrm{K}_{-}^{\prime}\right) \geq \mathrm{K}_{+}$.

Let the first of these relations be proved first. For the elements of $\boldsymbol{L}(\mathrm{XRYC})$ also belonging to $\boldsymbol{L}(\mathrm{YC})$, it is evident, since $\boldsymbol{L}(\mathrm{YC})<\mathrm{YC}$. In the remaining cases, let $\mathrm{X}$ be written under the form ALB. The concerned elements of $\boldsymbol{L}(\mathrm{XRYC})$ have the form BRYC. But BRYC $<\mathrm{XRYC}$ is equivalent to $\mathrm{B}<\mathrm{X}$, which is true because $\mathrm{BC} \in \boldsymbol{L}(\mathrm{XC})<\mathrm{XC}$.

The second relation is true for the elements of $\boldsymbol{R}(\mathrm{XRYC})$ belonging also to $\boldsymbol{R}(\mathrm{YC})$, and for YC itself (because it belongs to $\boldsymbol{R}(\mathrm{XRYC})$ ). The remainder of the elements are all superior to those of $\boldsymbol{R}(\mathrm{XC})$, themselves superior to YC from the hypotheses; this closes the demonstration.

\section{A.2.About the direct shadow.}

If there were such a couple of finite itineraries, called (UC,VC), it would fulfil the following conditions :

$$
\mathrm{XC}<\mathrm{U}<\mathrm{XRYC}
$$




$$
\begin{aligned}
& \mathrm{YC}>\mathrm{V}>\mathrm{YLXC} \\
& \boldsymbol{L}(\mathrm{U}, \mathrm{V})<\mathrm{XRYC} \\
& \boldsymbol{R}(\mathrm{U}, \mathrm{V})>\mathrm{YLXC}
\end{aligned}
$$

From (II.1) it is seen that $U=$ XRWC. But (II.1) and (II.4) imply that $\mathrm{YC}>\mathrm{W}>\mathrm{YLXC}$, so $\mathrm{W}=$ YLXRW', or U = XRYLXRW'. W' has then the same constraint as $\mathrm{W}$, so by induction one obtains $\mathrm{U}=(\mathrm{XRYL})^{\infty}$, which is not finite. Two conclusions are drawn :

(i) The rectangle of the form $[\mathrm{XC}, \mathrm{XRYC}] \times[\mathrm{YLXC}, \mathrm{YC}]$ does not contain any other finite contact.

(ii) The couple of infinite kneading sequences $\left((\mathrm{XRYL})^{\infty},(\mathrm{YLXR})^{\infty}\right)$ is admissible within this rectangle.

\section{A.3. Demonstration of topological chaos.}

Following a demonstration of [Zheng \& Lu, 1991], let $\mathrm{I}=$ $\mathrm{S}_{1} \mathrm{~S}_{2} \ldots \mathrm{S}_{\mathrm{n}} \ldots$, where $\mathrm{S}_{\mathrm{i}}$ is either XRYL $=\mathrm{E}_{1} \mathrm{~L}$ or $\mathrm{XRYRYL}=\mathrm{E}_{2} \mathrm{~L}$. To do so, it is enough to show that $\boldsymbol{L}(\mathrm{I})<\mathrm{XRYRYC}=\mathrm{E}_{2} \mathrm{C}$ and $\boldsymbol{R}(\mathrm{I})>\mathrm{YLXC}$.

First, the elements of $\boldsymbol{L}(\mathrm{I})$ having the form $\mathrm{S}_{\mathrm{i}} \mathrm{S}_{\mathrm{i}+1} \ldots$ are obviously inferior to XRYRYC. The proof remains to be done for the elements of $\boldsymbol{L}(\mathrm{I})$ of the form $\boldsymbol{L}\left(\mathrm{S}_{\mathrm{i}}\right) \mathrm{S}_{\mathrm{i}+1} \mathrm{~S}_{\mathrm{i}+2 \ldots} \ldots$ Two cases occur :

(i) $\boldsymbol{L}(\mathrm{I})=\boldsymbol{L}\left(\mathrm{E}_{1}\right) \mathrm{LS}_{\mathrm{i}+1} \mathrm{~S}_{\mathrm{i}+2} \ldots$. Here, $\boldsymbol{L}(\mathrm{I}) \leq \boldsymbol{L}\left(\mathrm{E}_{1}\right) \mathrm{C}$, itself equal to $\boldsymbol{L}\left(\mathrm{E}_{1} \mathrm{C}\right)$ and thus inferior to $\mathrm{E}_{1} \mathrm{C}$ and $a$ fortiori to $\mathrm{E}_{2} \mathrm{C}$.

(ii) $\boldsymbol{L}(\mathrm{I})=\boldsymbol{L}\left(\mathrm{E}_{2}\right) \mathrm{LS}_{\mathrm{i}+1} \mathrm{~S}_{\mathrm{i}+2} \ldots$. Here, $\boldsymbol{L}(\mathrm{I}) \leq \boldsymbol{L}\left(\mathrm{E}_{2}\right) \mathrm{C}=\boldsymbol{L}\left(\mathrm{E}_{2} \mathrm{C}\right) \leq$ $\mathrm{E}_{2} \mathrm{C}$.

The same demonstration is used for $\boldsymbol{R}(\mathrm{I})>$ YLXC, using the equivalent sequences $\mathrm{F}_{1} \mathrm{R}=\mathrm{YLXR}$ and $\mathrm{F}_{2} \mathrm{R}=\mathrm{YLXRYR}$. 


\section{Figure Captions.}

Fig. 1. First return map of the deformations $\delta_{n}$ (in \%), using values from the $a b$-initio calculations of [Cheng et al., 1990]. The plain line is a guess of the function $f$. The thick shaded lines of equations $\delta_{n}=\delta^{*}$ and $\delta_{n+1}$ $=\delta^{*}$ separate the domains of $\delta$ where the orientations $\mathrm{U}_{n}$ and $\mathrm{U}_{n+1}$ are $\mathbf{h}$ and $\mathbf{k}$.

Fig. 2. The example family of sawtooth maps as defined by relation (1).

Fig. 3. a) Two-parameter bifurcation diagram of family (1).

b) Zoom on the $[0.5,1] \times[0.5,1]$ region, where no stable steady state exists.

c) Detail of Fig. 3a) showing the structure of the crossing of two "tails".

Fig 4. a) Sawtooth map with attracting (stable) steady state.

b) Sawtooth map with attracting (stable) period-2 orbit.

Fig. 5. a) Illustration of rule $R 1$ : between two fish bodies of same degree of complexity and with a common nose lies another one.

b) Subharmonic cascade along the first diagonal.

c) Illustration of rule R2 : between two fish tails lies another fish.

Fig. 6. Zone of sensitivity to initial conditions (in black). Some nonsymmetrical points are artifacts due to very long chaotic transitory regimes.

Fig. 7. Plot of the function $g=T \circ \sigma \circ S$, numerical traduction of the shift function.

Fig. 8. Skeleton of the sawtooth maps drawn in the plane of kneading sequences. Crossings between two lines or solid contacts (thick dots) represent sawtooth maps with finite (superstable) kneading sequences.

Fig. 9. Iterated action of the transformation (F,LF ). 
The Sawtooth Map and the growth of SiC polytypes

Fig. 10. Location of known periodic polytypes of sequence length $\leq 50$ in the bifurcation diagram. 


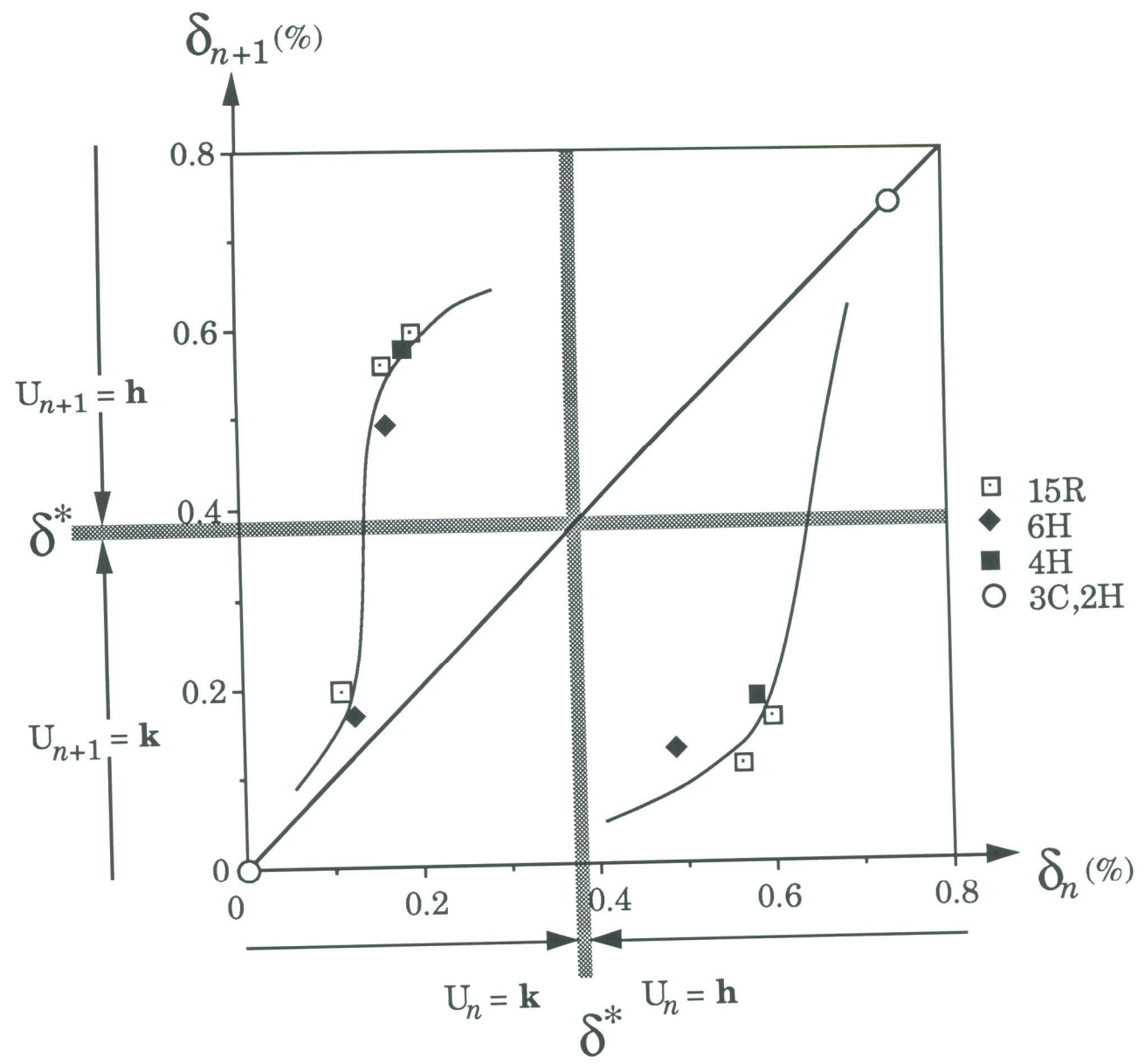

Fig.1.

G. L. VIGNOLES. 


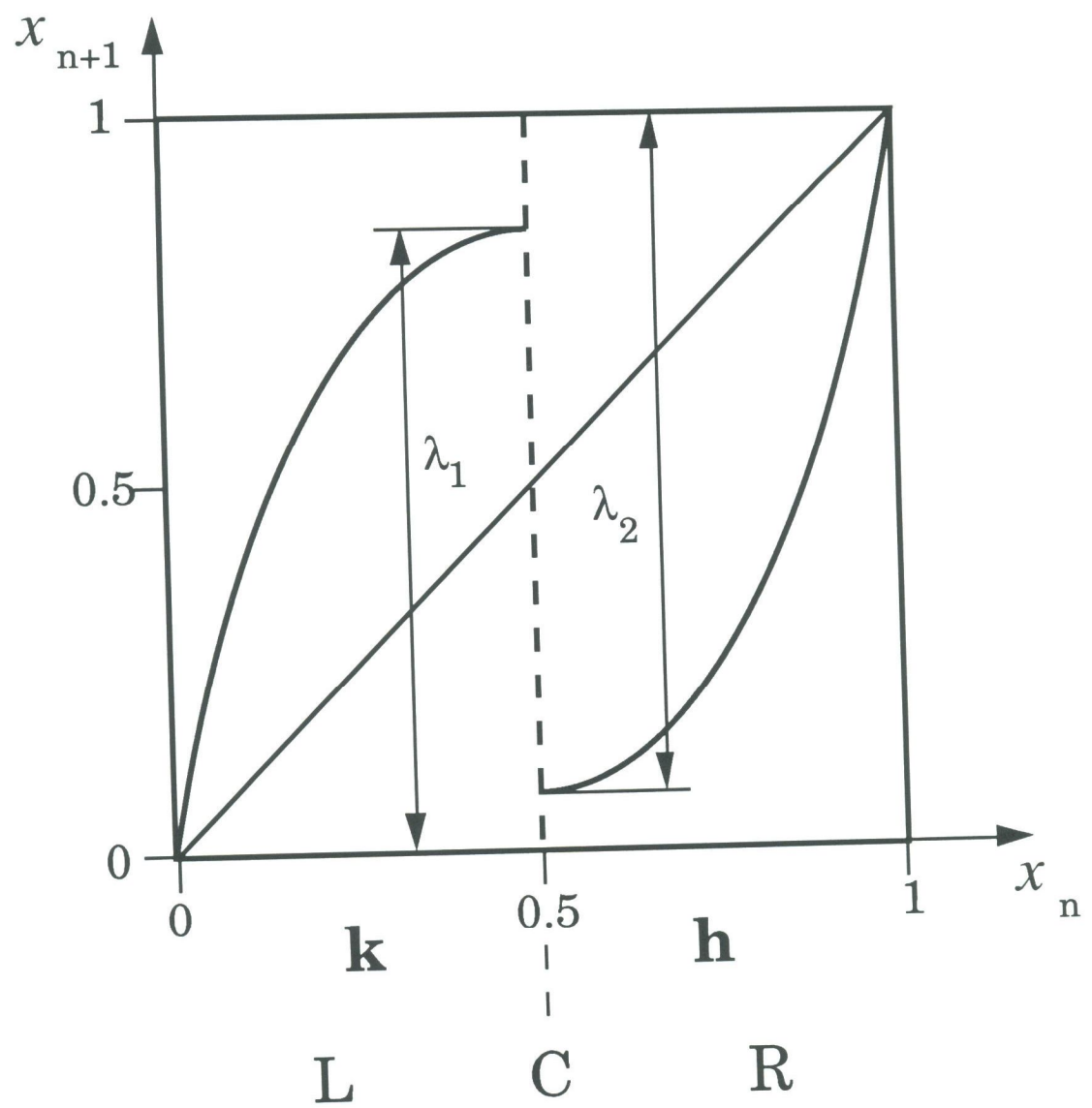

Fig. 2.

G. L. VIGNOLES. 

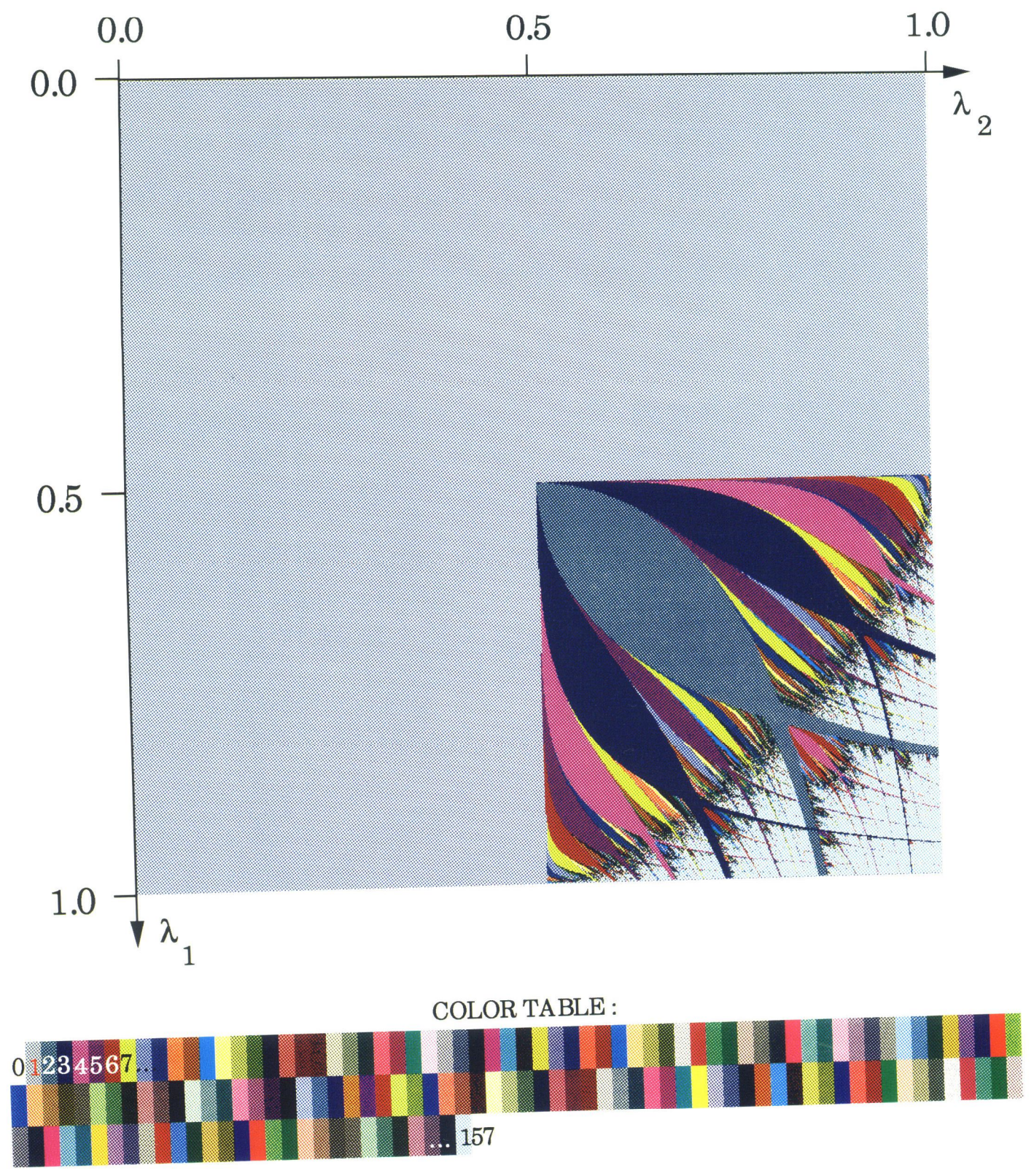

Fig. 3a)

G. L. VIGNOLES. 


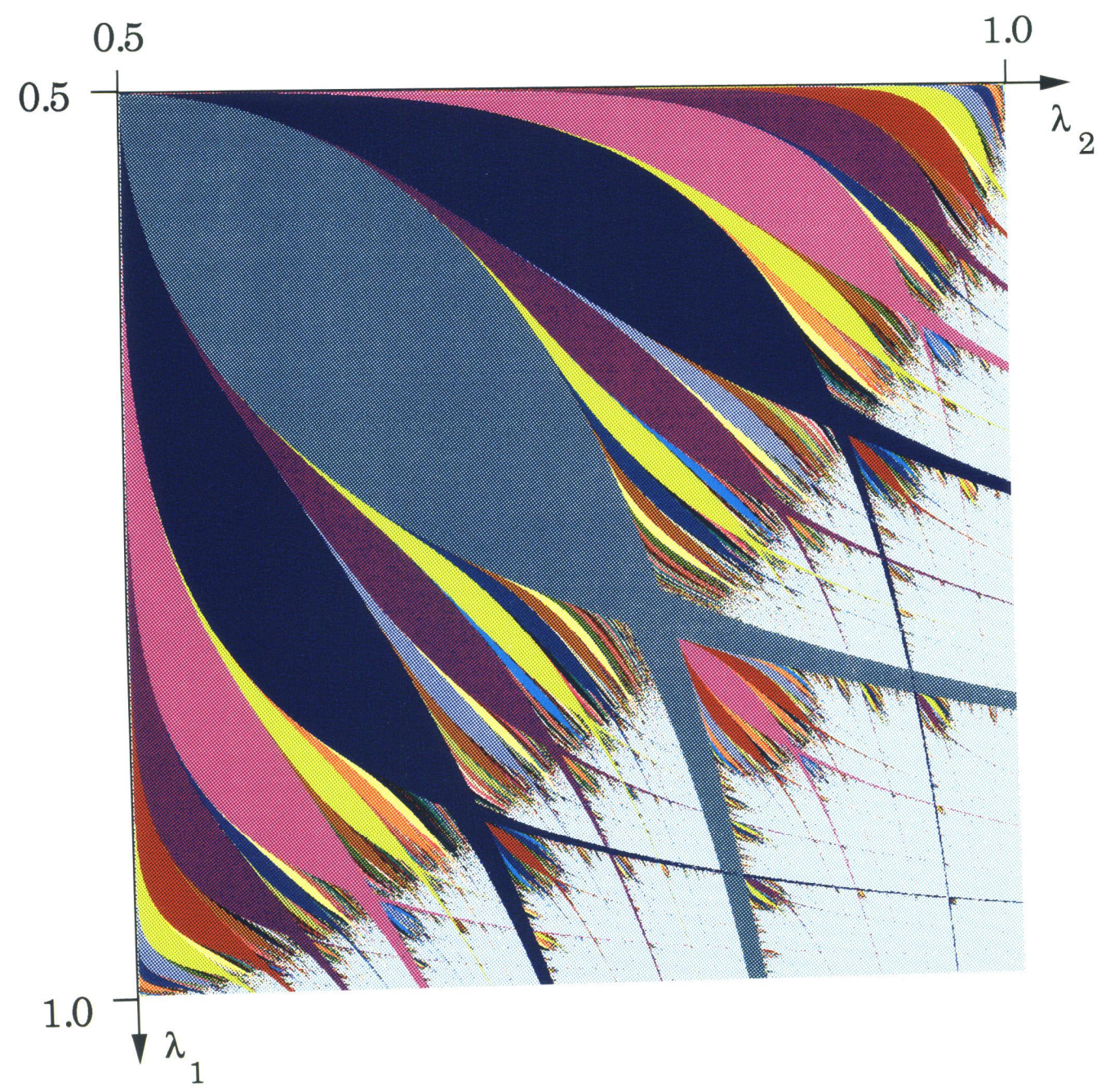

Fig. 3b)

G. L. VIGNOLES. 


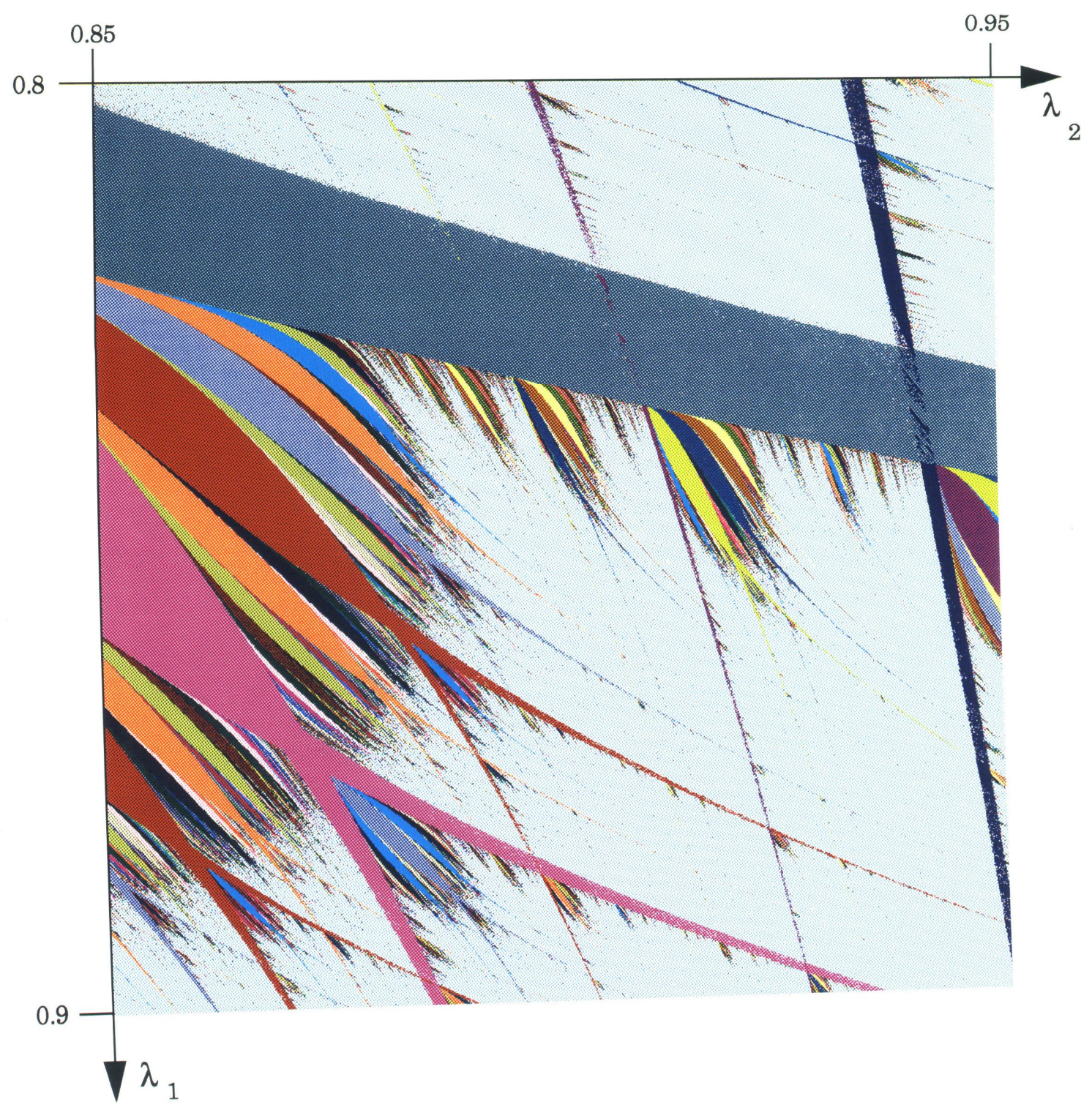

Fig. 3c)

G. L. VIGNOLES 


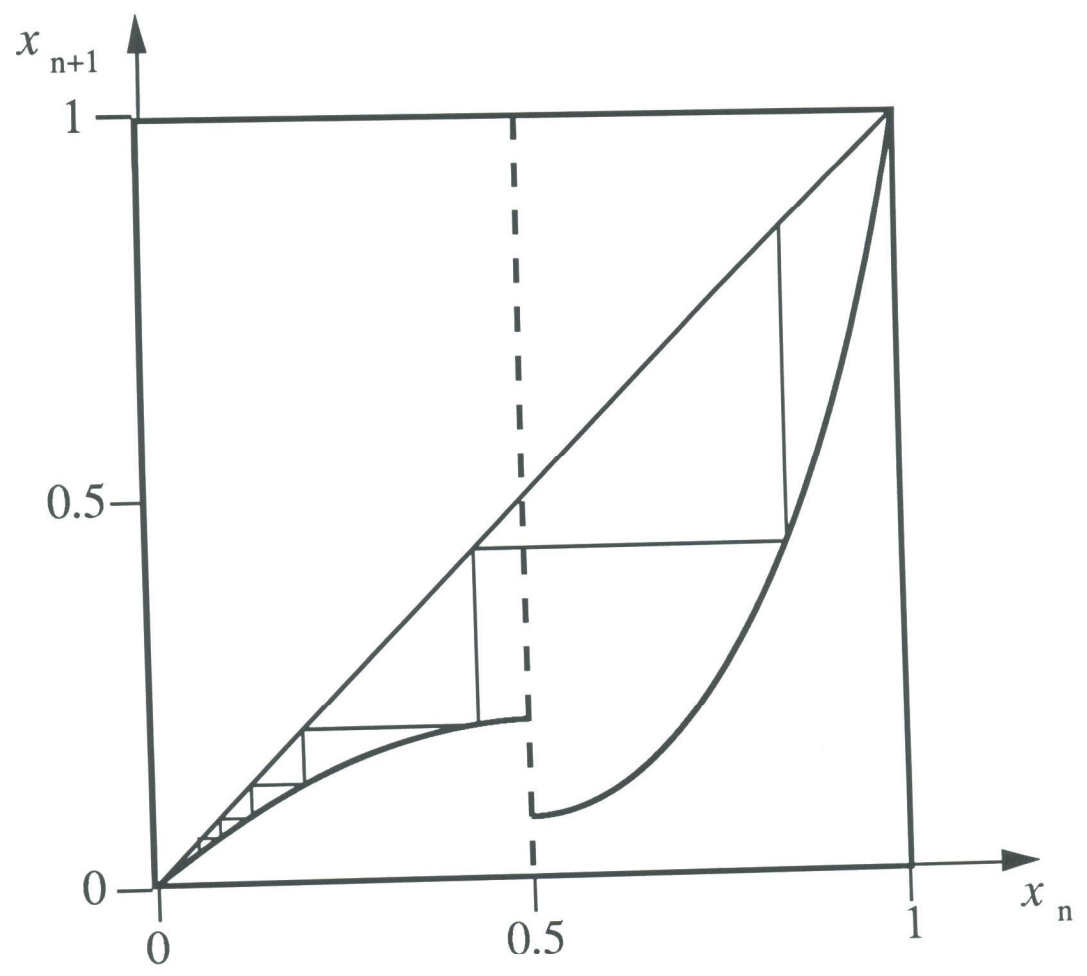

Fig. 4a).

G. L. VIGNOLES. 


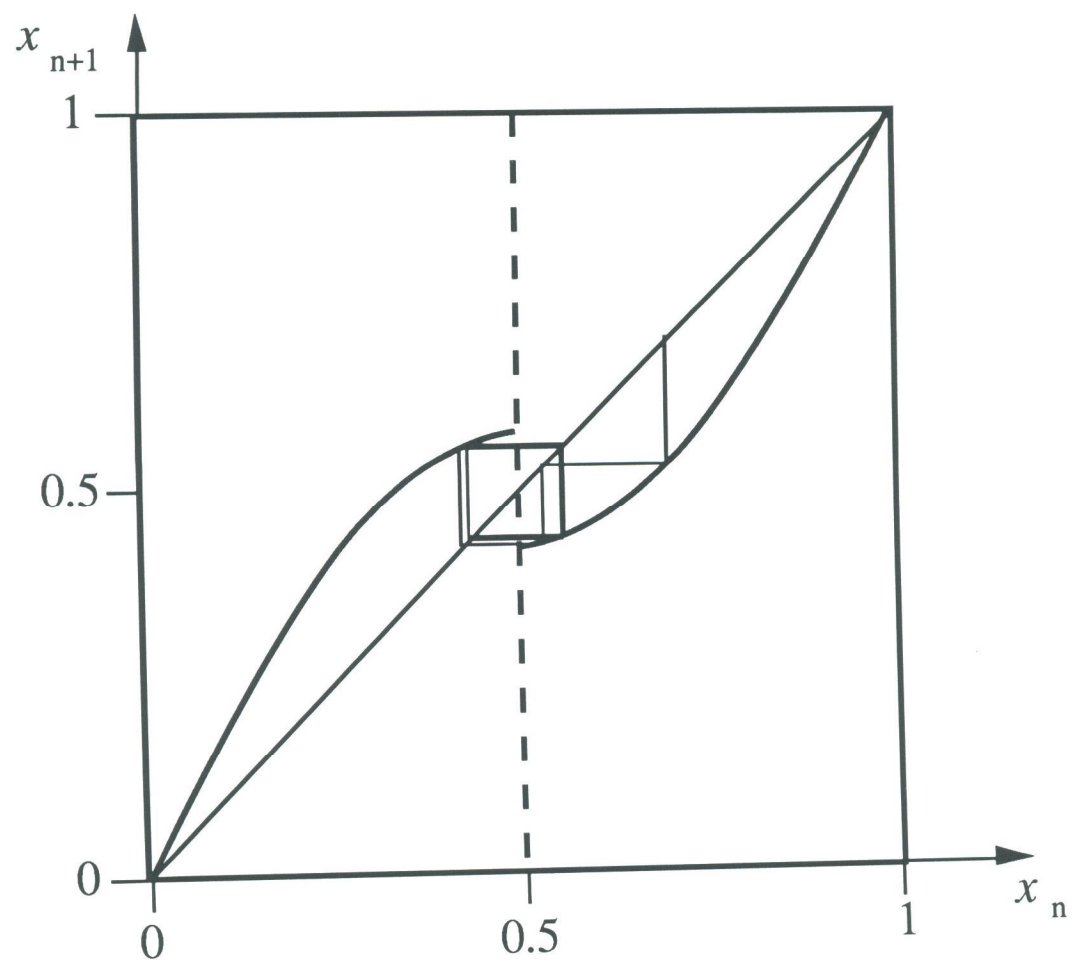

Fig. 4b).

G. L. VIGNOLES. 


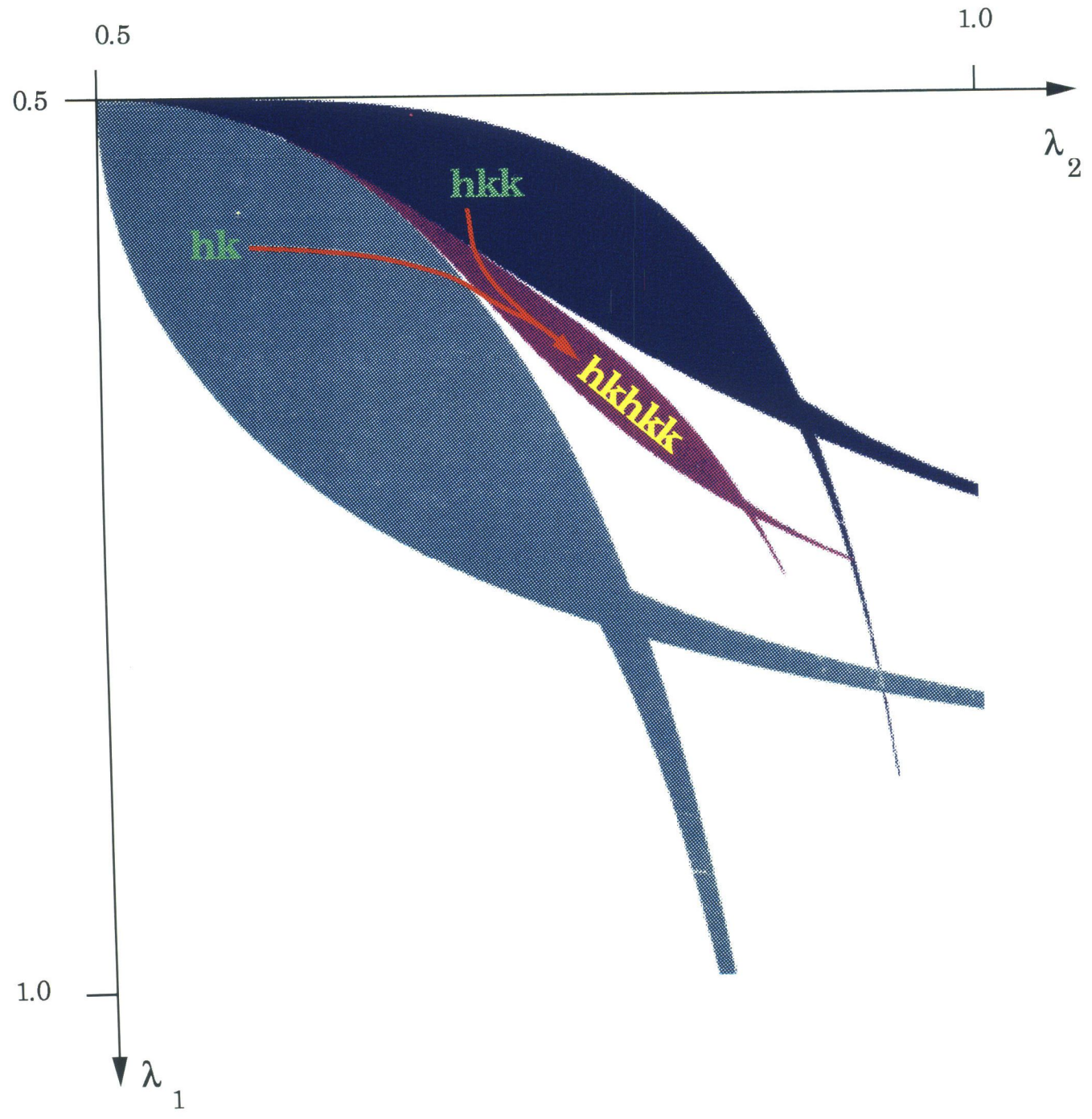

Fig. 5a)

G. L VIGNOLES. 


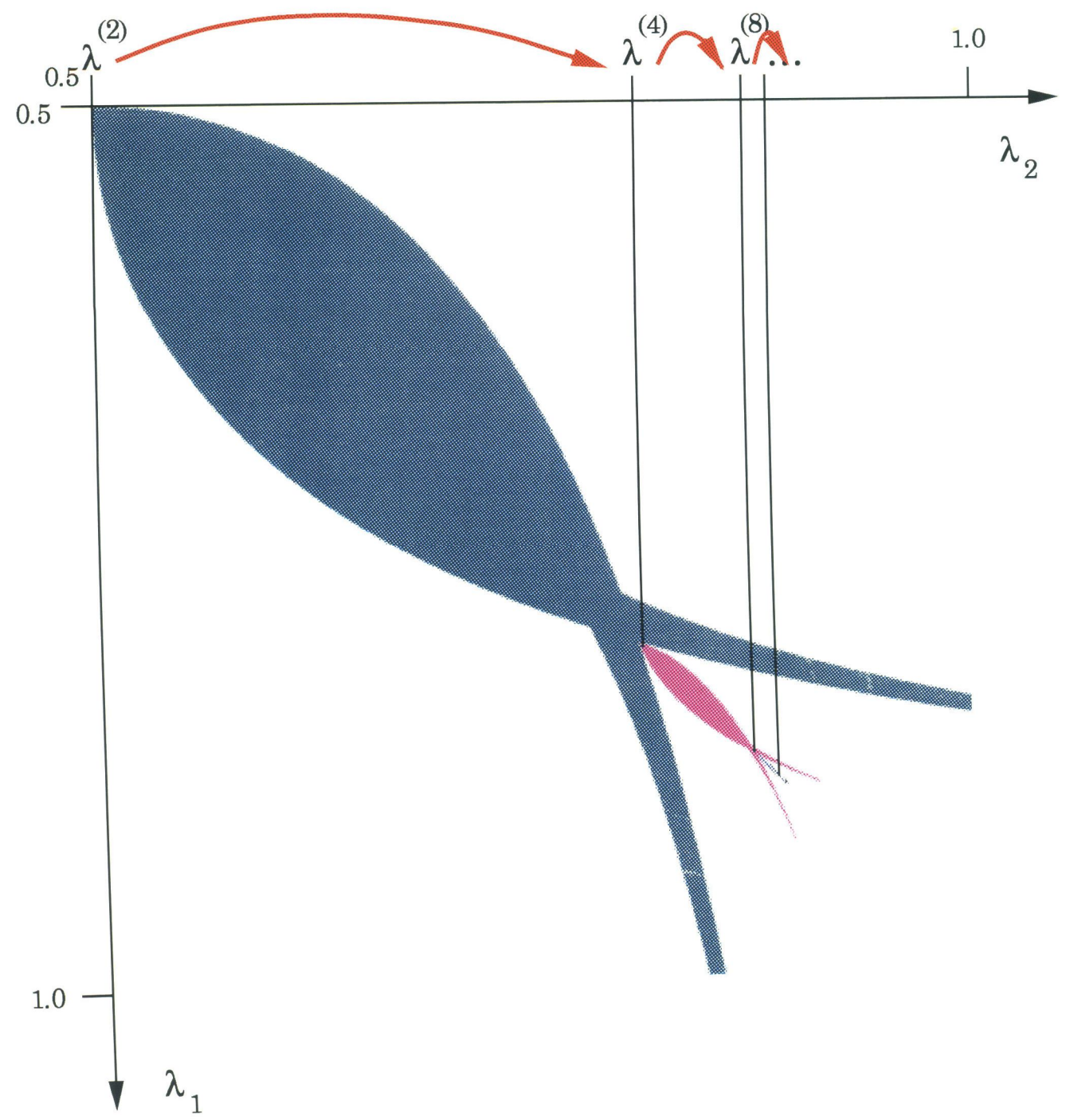

Fig. 5b)

G. L. VIGNOLES. 


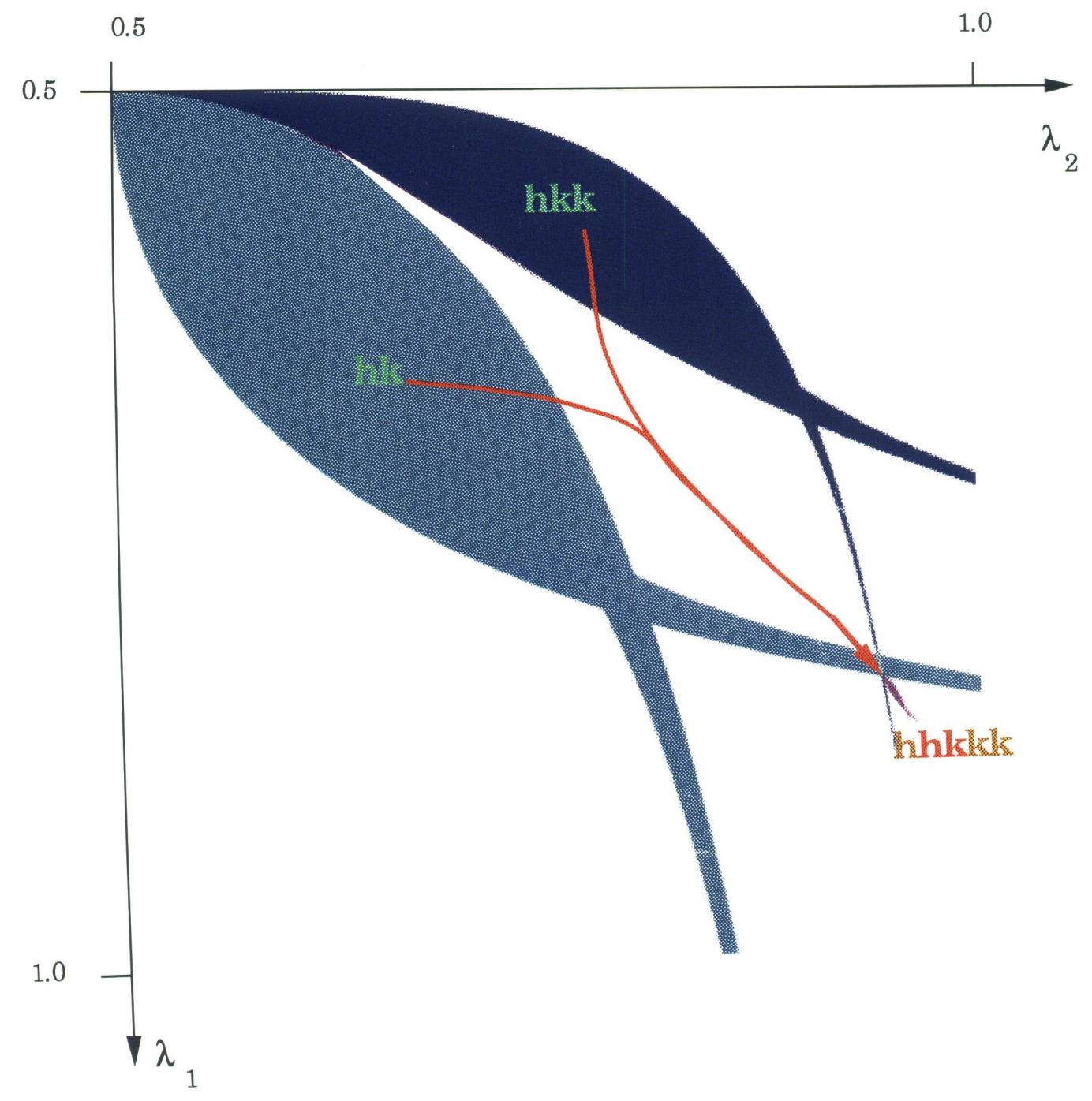

Fig. 5c)

G. L. VIGNOLES 


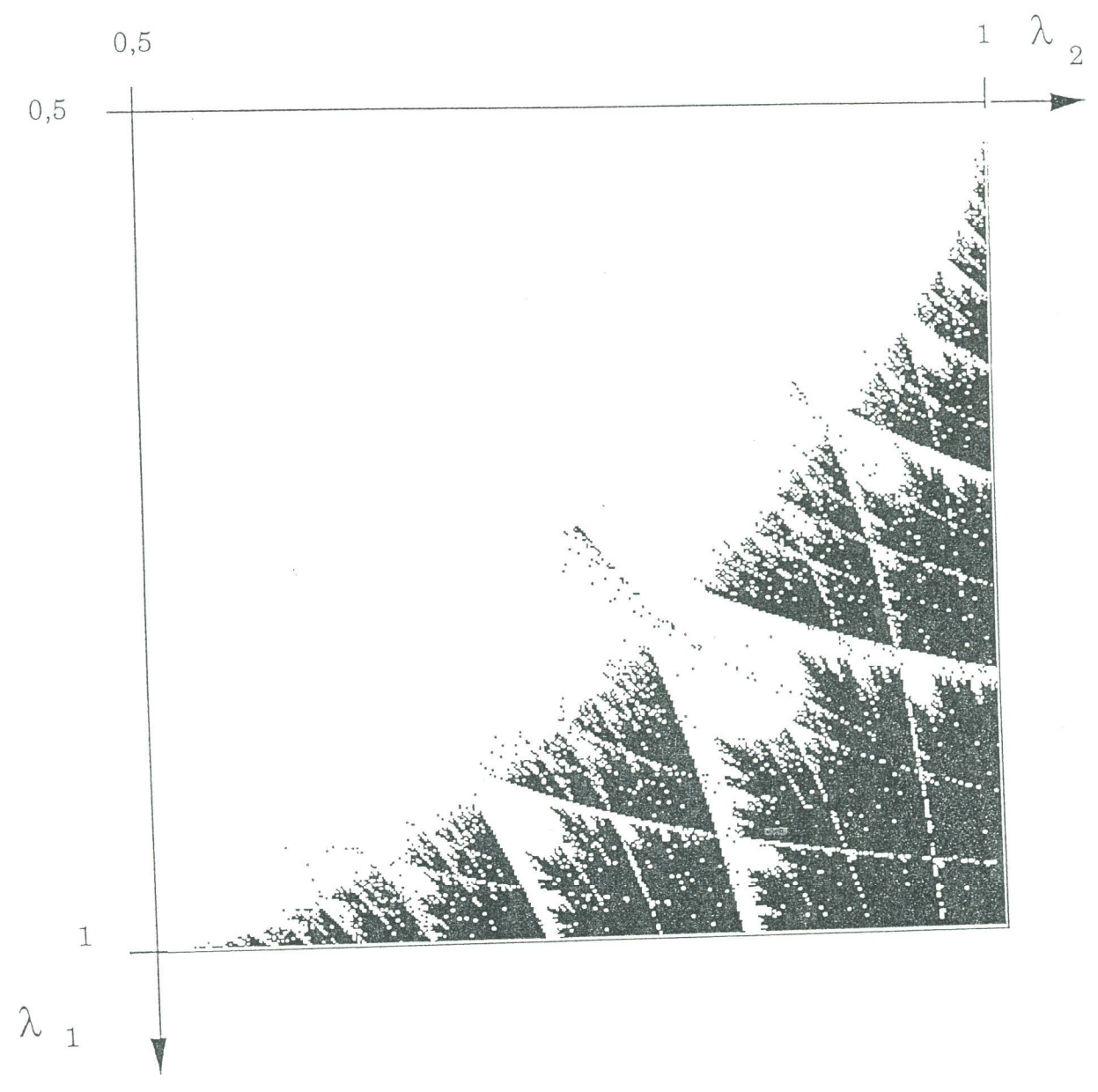

Fig. 6.

G. L. VIGNOLES. 


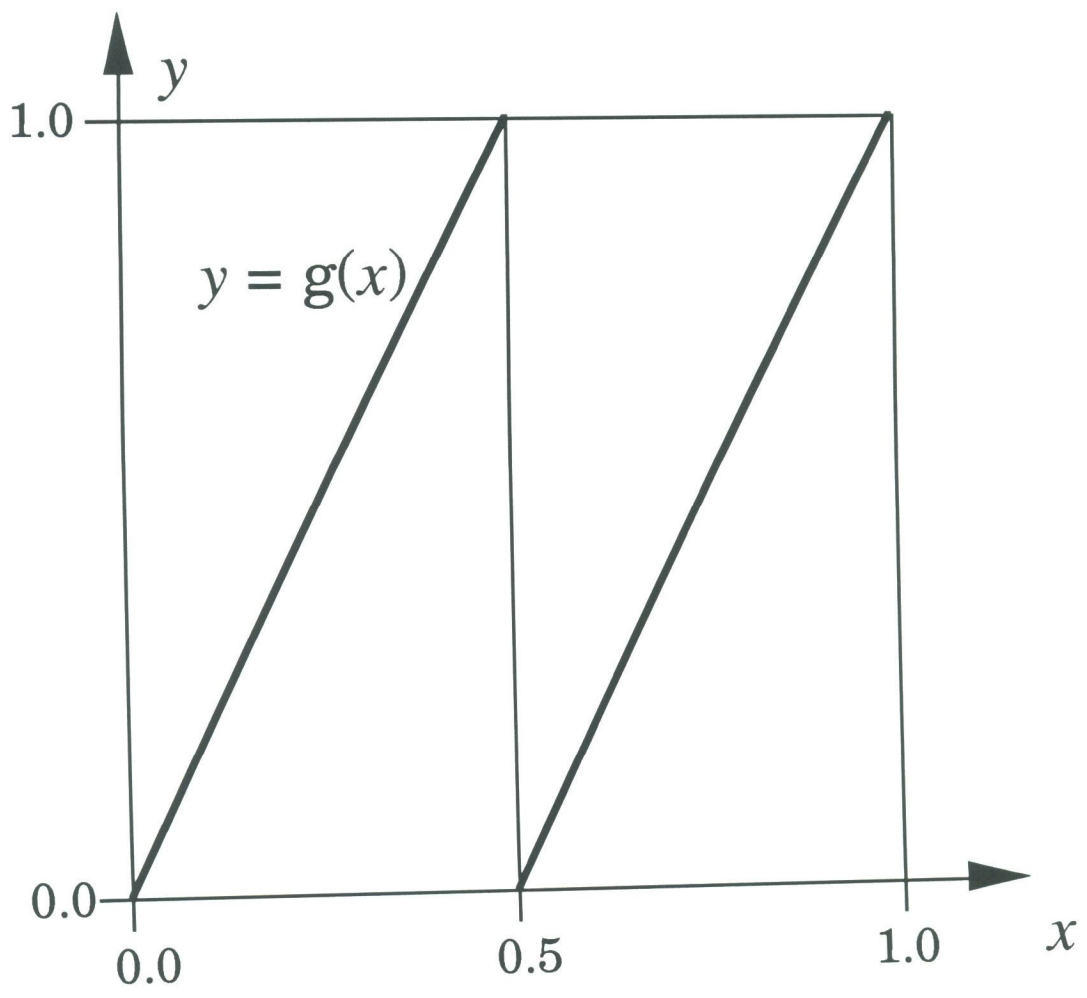

Fig. 7.

G. L. VIGNOLES, 

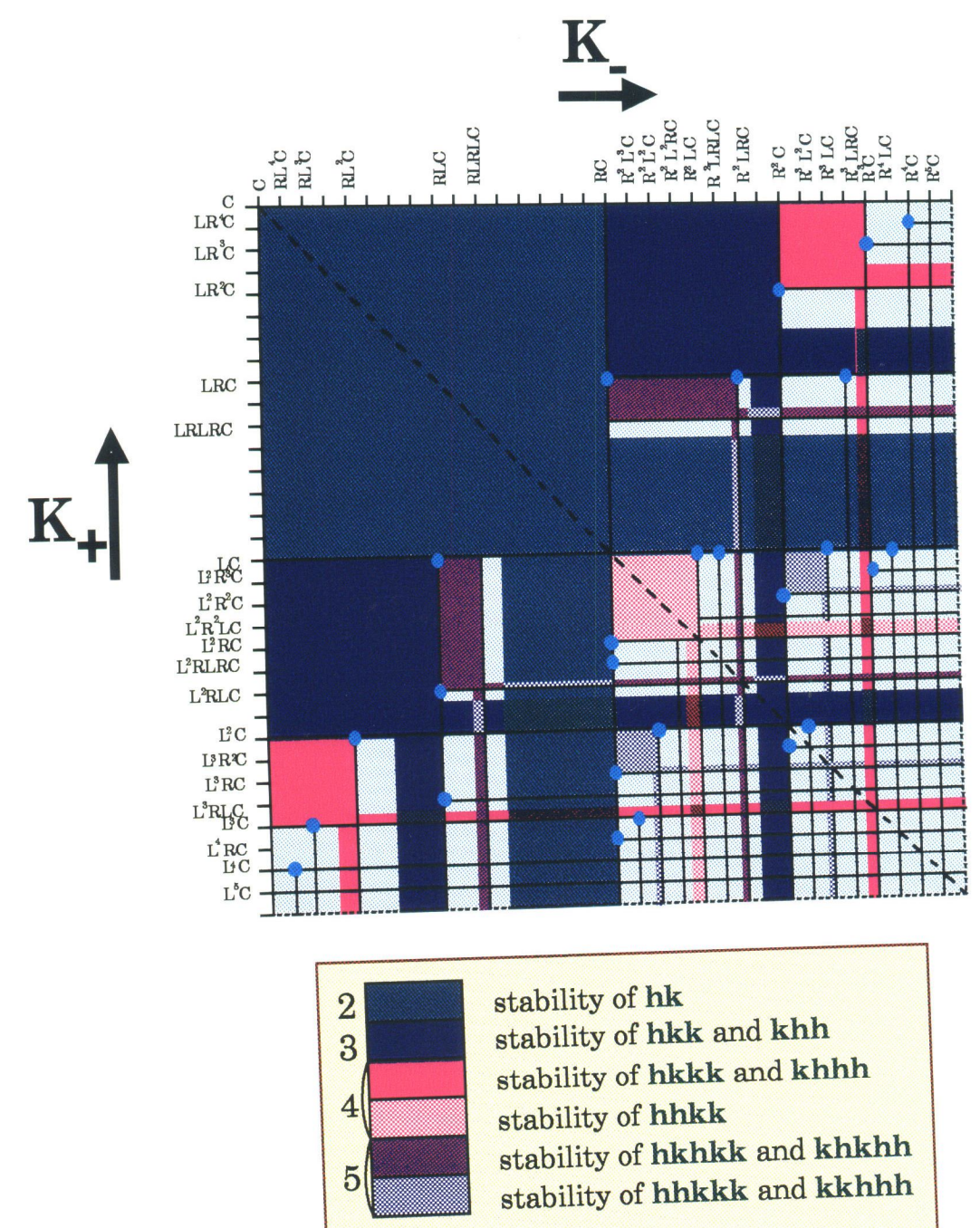

Fig. 8

G. L. VIGNOLES. 


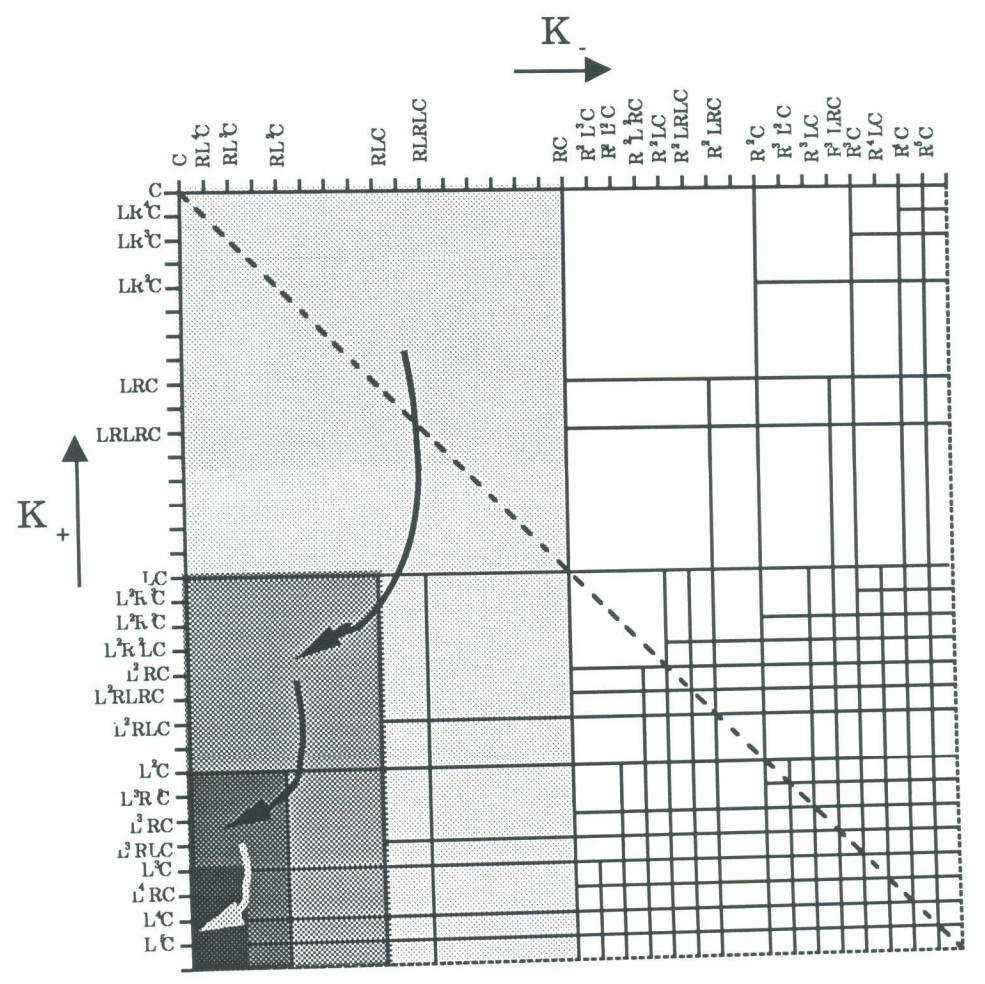

Fig. 9.

G. L. VIGNOLES. 


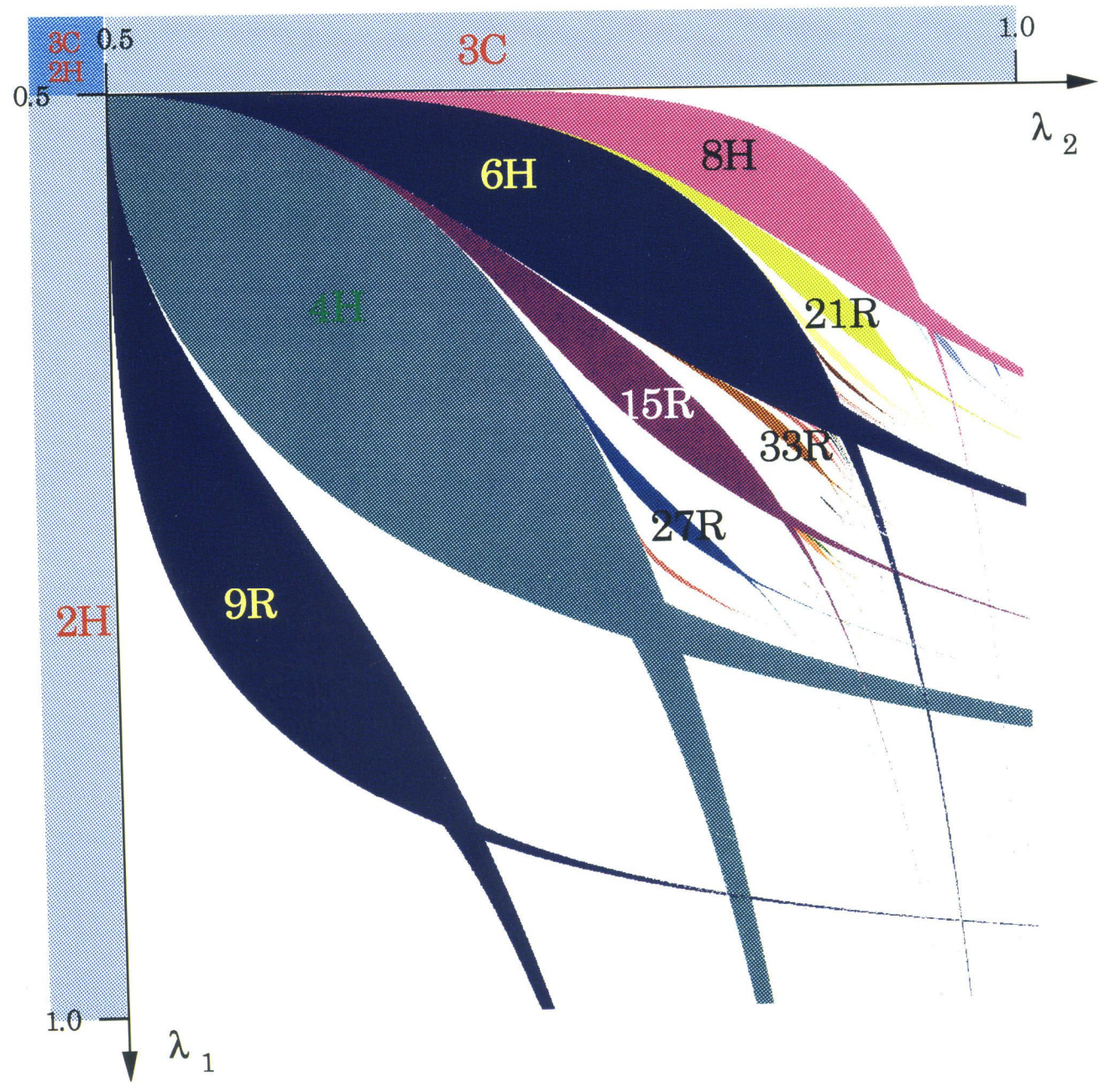

Fig. 10

G. L. VIGNOLES 